\title{
ON EXTREME VALUES OF NEHARI MANIFOLD METHOD VIA NONLINEAR RAYLEIGH'S QUOTIENT
}

\author{
YAVDAT IL'YASOV
}

\begin{abstract}
We study applicability conditions of the Nehari manifold method for the equation of the form $D_{u} T(u)-\lambda D_{u} F(u)=0$ in a Banach space $W$, where $\lambda$ is a real parameter. Our study is based on the development of the theory Rayleigh's quotient for nonlinear problems. It turns out that the extreme values of parameter $\lambda$ for the Nehari manifold method can be found through the critical values of a corresponding nonlinear generalized Rayleigh's quotient. In the main part of the paper, we provide some general results on this relationship. Applications are given to several types of nonlinear elliptic equations and systems of equations.
\end{abstract}

\section{INTRODUCTION}

The Nehari manifold method (NMM) 33, 34 is among the most powerful and widely used tool in the analysis of equations. Let us briefly describe it. Assume $W$ is a real Banach space, $\Phi_{\lambda}: W \rightarrow \mathbb{R}$ is a Fréchet-differentiable functional with derivative $D_{u} \Phi_{\lambda}$ and $\lambda \in \mathbb{R}$. Consider the equation of variational form

$$
D_{u} \Phi_{\lambda}(u)=0, \quad u \in W .
$$

The Nehari manifold associated with (园) is defined as the following subsets in $W$

$$
\mathcal{N}_{\lambda}:=\left\{u \in W \backslash 0: D_{u} \Phi_{\lambda}(u)(u)=0\right\} .
$$

Since any solution of (因) belongs to $\mathcal{N}_{\lambda}$, a natural idea to solve (図) is to consider the following constrained minimization problem

$$
\begin{aligned}
& \Phi_{\lambda}(u) \rightarrow \min \\
& D_{u} \Phi_{\lambda}(u)(u)=0, \quad u \in W .
\end{aligned}
$$

Suppose that there exists a local minimizer $u$ of this problem and $\Phi_{\lambda} \in C^{2}(U, \mathbb{R})$ for some neighbourhood $U \subset W$ of $u$, then by the Lagrange multiplier rule one has $\mu_{0} D_{u} \Phi_{\lambda}(u)+\mu_{1}\left(D_{u} \Phi_{\lambda}(u)+D_{u u} \Phi_{\lambda}(u)(u, \cdot)\right)=0$ for some $\mu_{0}, \mu_{1}$ such that $\left|\mu_{0}\right|+\left|\mu_{1}\right| \neq 0$. Testing this equality by $u$ we obtain $\mu_{1} D_{u u} \Phi_{\lambda}(u)(u, u)=0$. Hence, if $D_{u u} \Phi_{\lambda}(u)(u, u) \neq 0$, then we have successively $\mu_{1}=0, \mu_{0} \neq 0$ and therefore $D_{u} \Phi_{\lambda}(u)=0$. Thus, one has the following sufficient condition of the applicability of NMM

$$
D_{u u} \Phi_{\lambda}(u)(u, u) \neq 0 \text { for any } u \in \mathcal{N}_{\lambda} .
$$

2000 Mathematics Subject Classification. 35J50, 35J55, 35J60.

Key words and phrases. Nehari manifold; Rayleigh's Quotient; nonlinear elliptic equations; fibering method.

The author was partly supported by grants RFBR 13-01-00294-p-a, 14-01-00736-p-a. 
The feasibility of this condition often depends on parameter $\lambda$. Thus, we may suppose that there exists a set of the extreme values of Nehari manifold method $\left\{\lambda_{\min , i}, \lambda_{\max , i}\right\}_{i=1}^{\infty}$ such that the sufficient condition (卖) may hold only when $\lambda \in$ $\cup_{i}^{\infty}\left(\lambda_{\min , i}, \lambda_{\max , i}\right)$. This brings up a question of how to find these extreme values.

This question was a subject of investigations in 22, 23, 29]) where a method (the so-called spectral analysis by the fibering method [36, 37, 38]) of the finding variational principles corresponding to the extreme values of NMM has been introduced. Although this method has been applied to a number of problems (see e.g. [9, 12, 16, 18, 20, 21, 25, 27, 28]), it has certain disadvantages mainly due to its complexity. The complexity becomes especially notable when we are dealing with systems of equations (see e.g. [7]).

The aim of the present paper is to introduce a new approach to this problem. In order to indicate the principal idea of the approach, let us consider equation (因) in the following particular form

$$
D_{u} T(u)-\lambda D_{u} G(u)=0 .
$$

For simplicity, we assume that $D_{u} G(u)(u) \neq 0$ for any $u \in W \backslash 0$. Testing the equation by $u \in W$ and then solving it with respect to $\lambda=: r(u)$ we obtain the following functional

$$
r(u)=\frac{D_{u} T(u)(u)}{D_{u} G(u)(u)}, \quad u \in W \backslash 0,
$$

which we call the nonlinear generalized Rayleigh quotient (NG-Rayleigh quotient for short). Note that $u$ belongs $\mathcal{N}_{\lambda}$ if and only if it lies on the level set $r(u)=\lambda$. Using this fact we compute the following main identity

$$
D_{u} r(u)(u)=\frac{1}{D_{u} G(u)(u)} D_{u u}^{2} \Phi_{\lambda}(u)(u, u), \quad \forall u \in \mathcal{N}_{\lambda},
$$

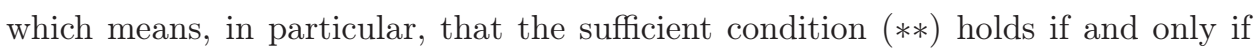
$D_{u} r(u)(u) \neq 0$. Notice that $D_{u} r(u)(u)=\partial r(t u) /\left.\partial t\right|_{t=1}$. These reasonings lead us to the main idea of our approach:

The extreme values of the Nehari manifold method can be found by means of studying the critical values of the fibered $N G$-Rayleigh quotient $\tilde{r}(t, u):=r(t u)$, $t \in \mathbb{R}^{+}, u \in W \backslash 0$.

This idea is consistent with the variational method of the finding eigenvalues of linear operators which has been introduced in 1869 by Weber [41] and then developed in works by Rayleigh, Fisher, Ritz, Courant (see e.g. [17, 19, 39, 42]). Indeed, by the minimax theorem the set of eigenvalues $\sigma:=\left\{\lambda_{1}, \ldots, \lambda_{n}\right\}$ of the $n \times n$ Hermitian matrix $A$ corresponds to the set of the critical values of Rayleigh's quotient $r(u):=\frac{\langle A u, u\rangle}{\langle u, u\rangle}$. Furthermore, if we formally consider the Nehari manifold minimization problem

$$
\left\{\begin{array}{l}
\langle A u, u\rangle-\lambda\langle u, u\rangle \rightarrow \min \\
\langle A u, u\rangle-\lambda\langle u, u\rangle=0, \quad u \in \mathbb{R}^{n} \backslash 0
\end{array}\right.
$$

then, as above, to apply it we should verify whether a priori solution of (1.4) will satisfy the equation $A u-\lambda u=0$. Thereby, we need to find the corresponding set of extreme values of NMM. The application of the above idea yields that this set coincides with the critical values of Rayleigh's quotient $r(u)$. Thus, in the linear case of (园), the problem of the finding extreme values of NMM is nothing else than the finding spectrum $\left\{\lambda_{1}, \ldots, \lambda_{n}\right\}$ of $A$, i.e. this is the eigenvalues problem. 
The paper is organized as follows. Section 2 contains some preliminaries on the

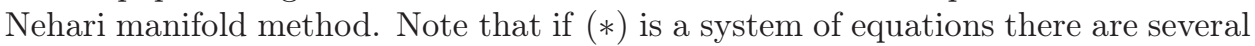
ways of introducing of the Nehari manifold. We discuss, in particular, the so-called vector and scalar Nehari manifold methods. Section 3 is devoted to the nonlinear generalized Rayleigh's quotient and its main properties. In Section 4, we introduce some basic extreme values of NMM by studying the critical values of the following functionals $\lambda(u):=\inf _{t>0} r(t u), \Lambda(u):=\sup _{t>0} r(t u)$ on $W \backslash 0$. In Section 5, we present several applications of the method where the extreme values of NMM can be expressed in explicit variational form. The aim of Section 7 is to show that NG-Rayleigh's quotient can be a useful tool itself in the analysis of equations. In particular, we prove using NG-Rayleigh's quotient a result on the existence of multiple solutions for an abstract equation and then, as a consequence, we obtain a novel result on the existence of multiple sign-constant solutions for a boundary value problem with a general convex-concave type nonlinearity and $p$-Laplacian.

\section{Notations}

We will denote by $W=W_{1} \times \ldots \times W_{n}$ the product of real Banach spaces $W_{i}$ with the norms $\|\cdot\|_{W_{i}}, i=1, \ldots, n$ and the norm $\|\cdot\|=\|\cdot\|_{W_{1}}+\ldots+\|\cdot\|_{W_{n}}$ in $W$.

To simplify the notation we write:

- $\dot{W}=\left(W_{1} \backslash 0\right) \times \ldots \times\left(W_{n} \backslash 0\right)$ and $\dot{\mathbb{R}}^{+}=\mathbb{R}^{+} \backslash 0$,

- $t:=\left(t_{1}, \ldots, t_{n}\right) \in \mathbb{R}^{n}$,

- $t \cdot u:=\left(t_{1} u_{1}, \ldots, t_{n} u_{n}\right), t u:=\left(t u_{1}, \ldots, t u_{n}\right),\langle t, u\rangle=\sum_{i=1}^{n} t_{i} u_{i}$, for $u \in W$, $t \in \mathbb{R}^{n}$

- $1_{n}=(1, \ldots, 1)^{T}$ and $0_{n}=(0, \ldots, 0)^{T}$ denote the vectors $1 \times n$ and $0 \times n$ in $\mathbb{R}^{n}$, respectively.

For $F \in C^{1}(W, \mathbb{R}), u \in W$, we write

- $\nabla_{u} F(u):=\left(D_{u_{1}} F(u), \ldots, D_{u_{n}} F(u)\right)^{T}$,

- $\nabla_{u} F(u)(v):=\left(D_{u_{1}} F(u)\left(v_{1}\right), \ldots, D_{u_{n}} F(u)\left(v_{n}\right)\right)^{T}, \quad v \in W$,

- $D_{u} F(u)(v):=\sum_{i=1}^{n} D_{u_{i}} F(u)\left(v_{i}\right)$,

where $D_{u_{i}} F(u)$ is the Frechet derivative with respect to $u_{i} \in W_{i}$ and $D_{u_{i}} F(u)\left(v_{i}\right)$ denotes the evaluation of $D_{u_{i}} F(u)$ at $v_{i} \in W_{i}, i=1,2, \ldots, n$.

For a given $F: W \rightarrow \mathbb{R}$, by the (vector) fibered map $\tilde{F}:\left(\mathbb{R}^{+}\right)^{n} \times W \rightarrow \mathbb{R}$ and the scalar fibered map $\tilde{F}^{s c}: \mathbb{R}^{+} \times W \rightarrow \mathbb{R}$ we mean the maps which are defined by $\tilde{F}(t, u):=F(t \cdot u)$ for $(t, u) \in\left(\mathbb{R}^{+}\right)^{n} \times W$ and $\tilde{F}^{s c}(t, u):=F(t u)$ for $(t, u) \in \mathbb{R}^{+} \times W$, respectively. We write

- $\nabla_{t} F(t \cdot u):=\left(\partial_{t_{1}} F(t \cdot u), \ldots, \partial_{t_{n}} F(t \cdot u)\right)^{T}$,

- $\nabla_{t} F(t \cdot u)(t):=\left(\partial_{t_{1}} F(t \cdot u) t_{1}, \ldots, \partial_{t_{n}} F(t \cdot u) t_{n}\right)^{T}$,

- $\partial F(t \cdot u) / \partial t:=\left\langle\nabla_{t} F(t \cdot u), t\right\rangle \equiv \sum_{i=1}^{n} \partial_{t_{i}} F(t \cdot u) t_{i}$.

\section{Preliminaries}

In the present paper, we shall deal with the $n$-dimenosional system of equations of the form

$$
\nabla_{u} \Phi_{\lambda}(u) \equiv \nabla_{u} T(u)-\lambda \nabla_{u} G(u)=0, \quad u \in \dot{W},
$$

where $\dot{W}=\Pi_{i=1}^{n}\left(W_{i} \backslash 0\right), T, G \in C^{1}(\dot{W}, \mathbb{R}), \lambda \in \mathbb{R}$ and $\Phi_{\lambda}(u)=T(u)-\lambda G(u)$. In the case $n=1$, we call (2.1) the scalar problem. We define the Nehari manifold 
associated with (2.1) as follows:

$$
\mathcal{N}_{\lambda}=\left\{u \in \dot{W}:\left.\nabla_{u} \Phi_{\lambda}(u)(u) \equiv \nabla_{t} \Phi_{\lambda}(t \cdot u)\right|_{t=1_{n}}=0\right\} .
$$

Then the corresponding Nehari manifold minimization problem is

$$
\left\{\begin{array}{l}
\Phi_{\lambda}(u) \rightarrow \min \\
\nabla_{u} \Phi_{\lambda}(u)(u)=0, \quad u \in \dot{W} .
\end{array}\right.
$$

We will say that $u_{0} \in \mathcal{N}_{\lambda}$ is a solution (or local minimizer) of (2.3) if there exists $\delta>0$ such that $\Phi_{\lambda}\left(u_{0}\right) \leq \Phi_{\lambda}\left(u_{0}\right)$ whenever $\left\|u-u_{0}\right\|_{W}<\delta, \quad u \in \mathcal{N}_{\lambda}$, and we denote by $\hat{\Phi}_{\lambda}$ the global minimization value in (2.3), i.e. $\hat{\Phi}_{\lambda}:=\inf \left\{\Phi_{\lambda}(u): u \in\right.$ $\mathcal{N}_{\lambda}$ \}. A solution $u \in \dot{W}$ of (2.1) is said to be ground state if there holds $\Phi_{\lambda}(u) \leq$ $\Phi_{\lambda}(w)$ for any solution $w \in \dot{W}$ of (2.1). Thus a global minimizer $u$ of (2.3) which satisfies equation (2.1) is a ground state.

From now on we make the following assumption:

$\nabla_{t} \Phi_{\lambda}(t \cdot u)$ is a map of class $C^{1}$ on $\left(\dot{\mathbb{R}}^{+}\right)^{n} \times \dot{W}$, i.e. $\nabla_{t} \tilde{\Phi}_{\lambda} \in C^{1}\left(\left(\dot{\mathbb{R}}^{+}\right)^{n} \times \dot{W}, \mathbb{R}^{n}\right)$.

Notice this assumption implies that the constraints $\nabla_{u} \Phi_{\lambda}(u)(u)$ in (2.3) is a manifolds of class $C^{1}$ on $\dot{W}$. Obviously any functional $\Phi_{\lambda} \in C^{2}(\dot{W}, \mathbb{R})$ satisfies to this assumption.

Let $u \in \dot{W}$. Consider the Jacobian matrix of vector-valued function $\Psi(t):=$ $\nabla_{u} \Phi_{\lambda}(t \cdot u)(t \cdot u)$, i.e.

$$
J_{t}\left(\nabla_{u} \Phi_{\lambda}(t \cdot u)(t \cdot u)\right)=\left[\frac{\partial}{\partial t_{1}} \Psi(t) \ldots \frac{\partial}{\partial t_{n}} \Psi(t)\right]
$$

or, component-wise:

$$
J_{t}\left(\nabla_{u} \Phi_{\lambda}(t \cdot u)(t \cdot u)\right)_{i, j}=\frac{\partial^{2}}{\partial t_{i} \partial t_{j}} \Phi_{\lambda}(t \cdot u) t_{j}+\delta_{i j} \frac{\partial}{\partial t_{j}} \Phi_{\lambda}(t \cdot u),
$$

where $\delta_{i j}=1$ if $i=j$ and $\delta_{i j}=0$ if $i \neq j$. To shorten the notation, we write $J\left(\nabla_{u} \Phi_{\lambda}(u)(u)\right):=\left.J_{t}\left(\nabla_{u} \Phi_{\lambda}(t \cdot u)(t \cdot u)\right)\right|_{t=1_{n}}$. Note that in the case $\Phi_{\lambda} \in C^{2}(\dot{W}, \mathbb{R})$ one has

$$
J\left(\nabla_{u} \Phi_{\lambda}(u)(u)\right)=\left(D_{u_{i} u_{j}}^{2} \Phi_{\lambda}(u)\left(u_{i}, u_{j}\right)+\delta_{i j} D_{u_{j}} \Phi_{\lambda}(u)\left(u_{j}\right)\right)_{\{1 \leq i, j \leq n\}} .
$$

Furthermore, if $u \in \mathcal{N}_{\lambda}$, then

$$
J\left(\nabla_{u} \Phi_{\lambda}(u)(u)\right)=\left(D_{u_{i} u_{j}}^{2} \Phi_{\lambda}(u)\left(u_{i}, u_{j}\right)\right)_{\{1 \leq i, j \leq n\}} .
$$

Let us prove

Lemma 2.1. Assume $\Phi_{\lambda} \in C^{1}(\dot{W}, \mathbb{R}), \nabla_{t} \tilde{\Phi}_{\lambda} \in C^{1}\left(\left(\dot{\mathbb{R}}^{+}\right)^{n} \times \dot{W}, \mathbb{R}^{n}\right)$. Suppose that there exists a solution $u_{0}$ of problem (2.3) such that

$$
\operatorname{det} J\left(\nabla_{u} \Phi_{\lambda}\left(u_{0}\right)\left(u_{0}\right)\right) \neq 0 .
$$

Then $u_{0}$ satisfies equation (2.1).

Proof. To simplify notation, we prove under the assumption $\Phi_{\lambda} \in C^{2}(\dot{W}, \mathbb{R})$. By Fritz John [30] conditions there exist the Lagrange multipliers $\mu_{0}, \mu_{1}, \ldots, \mu_{n}$ such that $\sum_{i=0}^{n}\left|\mu_{i}\right| \neq 0$ and

$$
\mu_{0} D_{u_{i}} \Phi_{\lambda}\left(u_{0}\right)+\sum_{j=1}^{n} \mu_{j}\left(D_{u_{i} u_{j}}^{2} \Phi_{\lambda}\left(u_{0}\right)\left(u_{0, j}, \cdot\right)+\delta_{i j} D_{u_{i}} \Phi_{\lambda}\left(u_{0}\right)\right)=0, \quad i=1, \ldots, n .
$$


Test these equations by $u_{0, i}, i=1, \ldots, n$, respectively. Then since $u_{0} \in \mathcal{N}_{\lambda}$, we obtain $J_{t}\left(\nabla_{u} \Phi_{\lambda}(u)(u)\right) \bar{\mu}=0$, where $\bar{\mu}:=\left(\mu_{1}, \ldots, \mu_{n}\right)^{T}$. However, in view of (2.5), this is possible only if $\mu_{1}=0, \ldots, \mu_{n}=0$. Hence, $\mu_{0} \neq 0$ and we obtain the required.

Let us mention that the Nehari manifold (2.2) is actually introduced by means of the vector fibered map $\tilde{\Phi}_{\lambda}(t, u):=\Phi_{\lambda}(t \cdot u),(t, u) \in\left(\mathbb{R}^{+}\right)^{n} \times W$. However, there is another approach which is based on the scalar fibered map $\tilde{\Phi}_{\lambda}^{s c}(t, u):=\Phi_{\lambda}(t u)$, $(t, u) \in \mathbb{R}^{+} \times W$. Indeed, let us introduce the scalar Nehari manifold

$$
\mathcal{N}_{\lambda}^{s c}=\left\{u \in W \backslash 0_{n}:\left.\partial_{t} \Phi_{\lambda}(t u)\right|_{t=1} \equiv D_{u} \Phi_{\lambda}(u)(u) \equiv \sum_{i=1}^{n} D_{u_{i}} \Phi_{\lambda}(u)\left(u_{i}\right)=0\right\} .
$$

Then the corresponding scalar Nehari manifold minimization problem is defined as follows

$$
\left\{\begin{array}{l}
\Phi_{\lambda}(u) \rightarrow \min \\
D_{u} \Phi_{\lambda}(u)(u) \equiv \sum_{i=1}^{n} D_{u_{i}} \Phi_{\lambda}(u)\left(u_{i}\right)=0, \quad u \in W \backslash 0_{n} .
\end{array}\right.
$$

Arguing as above, we have

Lemma 2.2. Assume $\Phi_{\lambda} \in C^{1}\left(W \backslash 0_{n}, \mathbb{R}\right), \partial_{t} \tilde{\Phi}_{\lambda} \in C^{1}\left(\dot{\mathbb{R}}^{+} \times\left(W \backslash 0_{n}\right), \mathbb{R}\right)$. Suppose that there exists a solution $u$ of problem (2.7) such that

$$
\left.\frac{\partial}{\partial t} D_{u} \Phi_{\lambda}(t u)(t u)\right|_{t=1} \neq 0 \text {. }
$$

Then $u$ satisfies equation (2.1).

Note that in the case $\Phi_{\lambda} \in C^{2}\left(W \backslash 0_{n}, \mathbb{R}\right)$ the condition (2.8) may be written as

$$
D_{u u}^{2} \Phi_{\lambda}(u)(u, u)=\sum_{i, j=1}^{n}\left(D_{u_{i} u_{j}}^{2} \Phi_{\lambda}(u)\left(u_{i}, u_{j}\right)+\delta_{i j} D_{u_{i}} \Phi_{\lambda}(u)(u)\right) \neq 0 .
$$

Let us remark that the assertion of Lemma 2.2 follows directly from Lemma 2.1 if we formally regard the system of equation (2.1) as a scalar equation.

In what follows, we call (2.3) and (2.7) the vector and scalar Nehari manifold method (NMM), respectively. Nevertheless, in most cases, we call (2.3) simply Nehari manifold method when no confusion can appears.

Remark 2.3. In the sequel, it will cause no confusion if we consider the method (2.7) as a particular case of the general approach (2.3). Moreover in the future mainly our reasoning (unless otherwise stated) will be carried out on problem (2.3), meaning that they are also valid for (2.7) as for the special case of (2.3).

Remark 2.4. It is worth noticing that $\nabla_{u} \Phi_{\lambda}(u)(u)=0$ and $\operatorname{det} J\left(\nabla_{u} \Phi_{\lambda}(u)(u)\right)=$ 0 for any $u \in W \backslash \dot{W}$, whereas $D_{u} \Phi_{\lambda}(u)(u)=0$ and $\left.\frac{\partial^{2}}{\partial t^{2}} \Phi_{\lambda}(t u)\right|_{t=1}=0$ if $u=0_{n}$. This is why in definitions (2.2), (2.6), we are setting $\mathcal{N}_{\lambda} \subset \dot{W}$, whereas $\mathcal{N}_{\lambda}^{s c} \subset$ $W \backslash 0_{n}$.

In the literature, the Nehari manifold minimization problem is sometimes considered in the following form (see e.g. [13, 23, 36, 37, 38])

$$
\left\{\begin{array}{l}
\Phi_{\lambda}(t \cdot v) \rightarrow \min \\
\nabla_{t} \Phi_{\lambda}(t \cdot v)=0_{n} \\
v \in S, t_{i}>0, \quad i=1,2, \ldots, n
\end{array}\right.
$$


where $S:=\left\{v \in W:\|v\|_{W}=1\right\}$ and the Nehari manifold is defined as follows

$$
\tilde{\mathcal{N}}_{\lambda}=\left\{(t, v) \in\left(\dot{\mathbb{R}}^{+}\right)^{n} \times S: \nabla_{t} \Phi_{\lambda}(t \cdot v)=0_{n}\right\} .
$$

It is readily seen that if $u$ is a solution of problem (2.3) such that (2.5) is satisfied, then $(t, v)$, where $t=1_{n}\|u\|, v=u /\|u\|$, is also a solution of (2.9) such that

$$
\operatorname{det}\left(J_{t}\left(\nabla_{t} \Phi_{\lambda}(t \cdot v)\right)\right) \neq 0 .
$$

The converse is also true.

Proposition 2.5. Assume $\Phi_{\lambda} \in C^{1}(\dot{W}, \mathbb{R}), \nabla_{t} \tilde{\Phi}_{\lambda} \in C^{1}\left(\left(\dot{\mathbb{R}}^{+}\right)^{n} \times \dot{W}, \mathbb{R}^{n}\right)$. Suppose $\left(t_{0}, v_{0}\right) \in \tilde{\mathcal{N}}_{\lambda}$ such that (2.11) is satisfied. Then there exists a neighbourhood $U\left(v_{0}\right)$ of $v_{0}$ in $S$ and a unique local $C^{1}$-map $t: U\left(v_{0}\right) \rightarrow \mathbb{R}^{n}$ such that $(t(v), v) \in \tilde{\mathcal{N}}_{\lambda}$ for all $v \in U\left(v_{0}\right)$ and $t\left(v_{0}\right)=t_{0}$.

Proof. By the assumption the determinant of the Jacobian of $\Psi(t):=\nabla_{t} \Phi_{\lambda}\left(t \cdot v_{0}\right)$ at the point $t=t_{0}$ is nonzero. Thus we may apply the Implicit Function Theorem to the functional $\psi(t, v):=\nabla_{t} \Phi_{\lambda}(t \cdot v)$ and the proof follows.

From this we are able to prove the following analogue of Lemma 2.1

Lemma 2.6. Assume $\Phi_{\lambda} \in C^{1}(\dot{W}, \mathbb{R}), \nabla_{t} \tilde{\Phi}_{\lambda} \in C^{1}\left(\left(\dot{\mathbb{R}}^{+}\right)^{n} \times \dot{W}, \mathbb{R}^{n}\right)$. Suppose that there exists a solution $\left(t_{0}, v_{0}\right)$ of problem (2.9) such that (2.11) holds. Then $u_{0}=t_{0} \cdot v_{0}$ satisfies equation (2.1).

Proof. Since (2.11) holds, we may apply Proposition 2.5. Thus there exists a neighbourhood $U\left(v_{0}\right) \subset S$ and a unique local $C^{1}$-map $t: U\left(v_{0}\right) \rightarrow \mathbb{R}^{n}$ such that $(t(v), v) \in \tilde{\mathcal{N}}_{\lambda}$ for all $v \in U\left(v_{0}\right)$ and $t\left(v_{0}\right)=t_{0}$. This implies that the function $\Phi_{\lambda}(t(v) v)$ constrained on $S$ attains a local minimum at point $v_{0} \in U\left(v_{0}\right)$. Consequently, by Fritz John conditions there exist the Lagrange multipliers $\mu_{0}, \mu_{1}$ such that $\left|\mu_{0}\right|+\left|\mu_{1}\right| \neq 0$ and

$$
\mu_{0} \nabla_{v} \Phi_{\lambda}\left(t\left(v_{0}\right) \cdot v_{0}\right)+\mu_{1} \nabla_{v}\left\|v_{0}\right\|=0 .
$$

Now testing this system of equations by $v_{0}$ we obtain as above in the proof of Lemma 2.1 that $\mu_{0} D_{v} \Phi_{\lambda}\left(t_{0} \cdot v_{0}\right)\left(v_{0}\right)+\mu_{1}=0$. Since $\nabla_{t} \Phi_{\lambda}\left(t_{0} \cdot v_{0}\right)=0_{n}$ we have $D_{v} \Phi_{\lambda}\left(t_{0} \cdot v_{0}\right)\left(v_{0}\right)=0$ and consequently $\mu_{1}=0$. This concludes the proof.

It is important to note that the definition of the Nehari manifold (2.2) $(\sqrt{2.10})$ and condition (2.5) (2.11) are invariant in the following sense

Proposition 2.7. Let $\psi:\left(\dot{\mathbb{R}}^{+}\right)^{n} \rightarrow\left(\dot{\mathbb{R}}^{+}\right)^{n}$ be $C^{1}$-map such that $\psi\left(1_{n}\right)=1_{n}$, $\operatorname{det}\left(\left.J(\psi(s))\right|_{s=1_{n}} \neq 0\right.$. Then $\left.\nabla_{s} \Phi_{\lambda}(\psi(s) \cdot u)\right|_{s=1_{n}}=0$ if and only if $\left.\nabla_{t} \Phi_{\lambda}(t \cdot u)\right|_{t=1_{n}}=$ 0 , and det $\left.J_{t}\left(\nabla_{s} \Phi_{\lambda}(\psi(s) \cdot u)(\psi(s) \cdot u)\right)\right|_{s=1_{n}} \neq 0$ if and only if det $J\left(\nabla_{u} \Phi_{\lambda}(u)(u)\right) \neq 0$.

Proof. It follows directly since $\left.\nabla_{s} \Phi_{\lambda}(\psi(s) \cdot u)\right|_{s=1_{n}}=\left.\left.J(\psi(s))\right|_{s=1_{n}} \nabla_{t} \Phi_{\lambda}(t \cdot u)\right|_{t=1_{n}}$ and $\left.\operatorname{det} J\left(\nabla_{s} \Phi_{\lambda}(\psi(s) \cdot u)(\psi(s) \cdot u)\right)\right|_{s=1_{n}}=\operatorname{det} J\left(\psi\left(1_{n}\right)\right) \operatorname{det} J\left(\nabla_{u} \Phi_{\lambda}(u)(u)\right)$. 


\section{Nonlinear Generalized Rayleigh's QUotient}

Denote $\mathcal{W}:=\left\{u \in W: D_{u} G(u)(u) \neq 0\right\}$. Evidently $0_{n} \notin \mathcal{W}$ and $\mathcal{W}$ is an open subset in $W$ if $G \in C^{1}(W, \mathbb{R})$. In the sequel, we always assume that $t \cdot u \in \mathcal{W}$ for any $u \in \mathcal{W}$ and $t \in \dot{\mathbb{R}}^{n}$.

The following functional plays a fundamental role in the present paper:

$$
r(u)=\frac{D_{u} T(u)(u)}{D_{u} G(u)(u)}, \quad u \in \mathcal{W} .
$$

We stress that $r(u)$ has been obtained by the following rule. Consider system of equations (2.1). Test these equations by $u_{i}, i=1, \ldots, n$, respectively. Summation then yields $D_{u} T(u)(u)-\lambda D_{u} G(u)(u)=0$. Now solving this equation with respect to $\lambda$ we obtain the functional (3.1). Notice that in the linear case of (2.1) $A u-\lambda u=0$, where $A$ is a $n \times n$ Hermitian matrix, the same rule gives the function

$$
r(u)=\frac{\langle A u, u\rangle}{\langle u, u\rangle}
$$

which is nothing else than ordinary Rayleigh's quotient 42, 41. For this reason, it makes sense to call (3.1) the nonlinear generalized Rayleigh's quotient ( $N G$ Rayleigh's quotient for short).

Note that $T, G \in C^{1}(W, \mathbb{R})$ implies $r(\cdot) \in C^{1}(\mathcal{W}, \mathbb{R})$. In what follows, we call $\tilde{r}(t, u):=r(t \cdot u)$ defined on $\left(\dot{\mathbb{R}}^{+}\right)^{n} \times \mathcal{W}$ a vector fibered $N G$-Rayleigh's quotient and $\tilde{r}^{s c}(t, u):=r(t u)$ defined on $\dot{\mathbb{R}}^{+} \times \mathcal{W}$ a scalar fibered $N G$-Rayleigh's quotient. For shorten notation, we will call $\tilde{r}(t, u)$ simply fibered NG-Rayleigh's quotient when no confusion can appears. Clearly, $\tilde{r}(\cdot, u) \in C^{1}\left(\left(\dot{\mathbb{R}}^{+}\right)^{n}, \mathbb{R}\right)$ and $\tilde{r}^{s c}(\cdot, u) \in C^{1}\left(\dot{\mathbb{R}}^{+}, \mathbb{R}\right)$ for every $u \in \mathcal{W}$.

Our basic assumption on NG-Rayleigh's quotient $r(u)$ is the following: For every fixed $u \in \mathcal{W}$ and $a_{n} \in\left(\dot{\mathbb{R}}^{+}\right)^{n} \backslash \dot{\mathbb{R}}^{n}$, there exists $\lim _{t \rightarrow a_{n}} r(t \cdot u)=\hat{r}\left(a_{n}, u\right)$, where $\left|\hat{r}\left(a_{n}, u\right)\right| \leq \infty$, thereby there exists a continuation of the fibered mapping $\tilde{r}(t, u):=$ $r(t \cdot u)$ to $\left(\dot{\mathbb{R}}^{+}\right)^{n} \times \mathcal{W}$ by setting $\tilde{r}\left(a_{n}, u\right):=\hat{r}\left(a_{n}, u\right)$ for each $u \in \mathcal{W}$ and $a_{n} \in$ $\left(\dot{\mathbb{R}}^{+}\right)^{n} \backslash \dot{\mathbb{R}}^{n}$. In the scalar case, we make the similar assumption: For every fixed $u \in \mathcal{W}$, there exists $\lim _{t \rightarrow 0} \tilde{r}^{s c}(t, u)=\tilde{r}^{s c}(0, u)$, thereby there exists a continuation of $\tilde{r}^{s c}(t, u)$ on $\mathbb{R}^{+} \times \mathcal{W}$.

In the next corollaries we collected some basic properties of $r(u)$.

Corollary 3.1. For any $u \in \mathcal{W}$ and $t \in\left(\dot{\mathbb{R}}^{+}\right)^{n}$ there holds

(i) $r(t \cdot u)=\lambda$ if and only if $\partial \Phi_{\lambda}(t \cdot u) / \partial t=0$;

(ii) if $t \cdot u \in \mathcal{N}_{\lambda}$, then $\lambda=r(t \cdot u)$.

Furthermore, if $D_{u} G(t \cdot u)(t \cdot u)>0\left(D_{u} G(t \cdot u)(t \cdot u)<0\right)$ for $u \in \mathcal{W}$ and $t \in\left(\dot{\mathbb{R}}^{+}\right)^{n}$, then:

(iii) $r(t \cdot u)>\lambda$ if and only if $\partial \Phi_{\lambda}(t \cdot u) / \partial t>0$;

(iv) $r(t \cdot u)<\lambda$ if and only if $\partial \Phi_{\lambda}(t u) / \partial t<0$.

Proof. To obtain the proof it is sufficient to note that $\lambda=r(t \cdot u)$ is nothing else than the root of the equation

$$
\partial \Phi_{\lambda}(t \cdot u) / \partial t \equiv\left\langle\nabla_{t} \Phi_{\lambda}(t \cdot u), t\right\rangle=\left\langle\nabla_{t} T(t \cdot u), t\right\rangle-\lambda\left\langle\nabla_{t} G(t \cdot u), t\right\rangle=0 .
$$

For the scalar fibered NG-Rayleigh's quotient, in addition to Corollary 3.1 we have 
Corollary 3.2. Consider the scalar Nehari manifold (2.6). Then for $u \in \mathcal{W}$ and $t>0$ there holds:

(i) $t u \in \mathcal{N}_{\lambda}^{s c}$ if and only if $\lambda=r(t u)$.

Furthermore, if $D_{u} G(t u)(t u)>0\left(D_{u} G(t u)(t u)<0\right)$ for $u \in \mathcal{W}$ and $t \in \mathbb{R}^{+}$, then:

(ii) $\partial r(t u) / \partial t<0$ if and only if $\partial^{2} \Phi_{\lambda}(t u) / \partial t^{2}<0\left(\partial^{2} \Phi_{\lambda}(t u) / \partial t^{2}>0\right)$;

(iii) $\partial r(t u) / \partial t>0$ if and only if $\partial^{2} \Phi_{\lambda}(t u) / \partial t^{2}>0\left(\partial^{2} \Phi_{\lambda}(t u) / \partial t^{2}<0\right)$.

Proof is evident.

Note that in the scalar case if $\mathcal{W}=W \backslash 0$, then $u \in \mathcal{N}_{\lambda}^{s c}$ if and only if $\lambda=r(u)$. Therefore in this case the Nehari manifold can be defined also as follows

$$
\mathcal{N}_{\lambda}^{s c}=\{u \in W \backslash 0: r(u)=\lambda\}, \quad \lambda \in \mathbb{R},
$$

and the minimization problem (2.3) for $\lambda \in \mathbb{R}$ can be written in the following equivalent form

$$
\left\{\begin{array}{l}
\Phi_{\lambda}(u) \rightarrow \min \\
r(u)=\lambda, \quad u \in W \backslash 0 .
\end{array}\right.
$$

Let $u \in \mathcal{W}$. We call $t_{u} \in\left(\dot{\mathbb{R}}^{+}\right)^{n}$ the extremal point of $r(t \cdot u)$ if the function $r(t \cdot u)$ attains at $t_{u}$ its local maximum or minimum on $\left(\dot{\mathbb{R}}^{+}\right)^{n}$. If $\nabla_{t} r\left(t_{u} \cdot u\right)=0_{n}$, then $t_{u}$ is said to be a critical point of $r(t \cdot u)$ and $\lambda=r\left(t_{u} \cdot u\right)$ is called the critical value.

Let $u \in \mathcal{W}$ and $t \in\left(\dot{\mathbb{R}}^{+}\right)^{n}$. Then by (3.1) we have

$$
\nabla_{t} r(t \cdot u)=\frac{\left.J_{t}\left(\nabla_{u} \Phi_{\lambda}(t \cdot u)(t \cdot u)\right) 1_{n}\right|_{\lambda=r(t \cdot u)}}{D_{u} G(t \cdot u)(t \cdot u)} .
$$

Proposition 3.3. For any $u \in \mathcal{W}$ and $t \in\left(\dot{\mathbb{R}}^{+}\right)^{n}$ such that $t \cdot u \in \mathcal{N}_{\lambda}$ with $\lambda=r(t \cdot u)$, there holds

(i) $\nabla_{t} r(t \cdot u)=0_{n}$ implies $\operatorname{det} J_{t}\left(\nabla_{u} \Phi_{\lambda}(t \cdot u)(t \cdot u)\right)=0$;

(ii) $J_{t}\left(\nabla_{u} \Phi_{\lambda}(t \cdot u)(t \cdot u)\right) 1_{n}=0_{n}$ implies $\nabla_{t} r(t \cdot u)=0$.

Proof is evident.

Observe, in the case of scalar NMM, since $\left.D_{u} \Phi_{\lambda}(t u)(u)\right|_{\lambda=r(t u)}=0$ for any $u \in \mathcal{W}$, formula (3.4) can be written as follows

$$
\partial_{t} r(t u)=\left.\frac{1}{D_{u} G(t u)(t u)} D_{u u}^{2} \Phi_{\lambda}(t u)(t u, u)\right|_{\lambda=r(t u)} .
$$

Hence, in this case, $\partial_{t} r(t u)=0$ for $t>0$ if and only if $D_{u u}^{2} \Phi_{\lambda}(t u)(t u, u)=0$, $\lambda=r(t u)$.

We will make the following assumption:

(A) If $u \in \mathcal{W}, t \in\left(\dot{\mathbb{R}}^{+}\right)$and $t \cdot u \in \mathcal{N}_{r(t \cdot u)}$, then the equality

$$
\left.\operatorname{det} J_{t}\left(\nabla_{u} \Phi_{\lambda}(t \cdot u)(t \cdot u)\right)\right|_{\lambda=r(t \cdot u)}=0
$$

implies that $t$ is an extremal point of $r(t \cdot u)$.

Remark 3.4. In the case of scalar NMM, in view of (3.5) assumption (A) means that $r(t u)$ for $u \in \mathcal{W}$ has no critical points in $\mathbb{R} \backslash 0$ other than extremal. 
In general position, one may assume that for every $u \in \mathcal{W}$ the function $r(t$. $u$ ) has a countable (or finite) set of extremal points $t_{u}^{1}, t_{u}^{2} \ldots$, which determine the continuous mappings $t_{(\cdot)}^{i}: \dot{W} \rightarrow\left(\dot{\mathbb{R}}^{+}\right)^{n}, i=1,2, \ldots$ so that there exist the functionals $\lambda_{i}(u):=r\left(t_{u}^{i} \cdot u\right), u \in \dot{W}, i=1,2, \ldots$ Consider

$$
\lambda_{\min , i}=\inf _{u \in \mathcal{W}} \lambda_{i}(u), \quad \lambda_{\max , i}=\sup _{u \in \mathcal{W}} \lambda_{i}(u), \quad i=1,2, \ldots
$$

Our basic conjecture is that the set of extreme values of Nehari manifold method contains in the set $\sigma:=\left\{\lambda_{\min , i}, \lambda_{\max , i}\right\}_{i=1}^{\infty}$.

Let us stress that $\lambda_{\min , i}$ and $\lambda_{\max , i}$ are 0 -homogeneous functionals on $\mathcal{W}$, i.e. $\lambda_{\min , i}(s u)=\lambda_{\min , i}(u), \lambda_{\max , i}(s u)=\lambda_{\max , i}(u)$ for any $s \in\left(\dot{\mathbb{R}}^{+}\right)^{n}$ and $u \in \mathcal{W}$. Note that ordinary Rayleigh's quotient $r(u)=\frac{\langle A u, u\rangle}{\langle u, u\rangle}$ possess the similar property.

In the present paper, to find extreme values of NMM, we mainly deal with the following functionals

$$
\lambda(u):=\inf _{t \in\left(\mathbb{R}^{+}\right)^{n}} r(t \cdot u), \quad \Lambda(u):=\sup _{t \in\left(\mathbb{R}^{+}\right)^{n}} r(t \cdot u) \text { on } \mathcal{W} .
$$

Notice that if there exist the extremal points $t_{u, \max }, t_{u, \min }$, where the function $\tilde{r}(\cdot, u)$ attains its global maximum and minimum values, respectively, then $\lambda(u)=$ $r\left(t_{u, \min } \cdot u\right), \Lambda(u)=r\left(t_{u, \max } \cdot u\right)$.

Remark 3.5. In view of Proposition 2.7, all of the above statements (Corollaries 3.1, 3.2, Proposition 3.3 etc.) still hold after making a change of variable $t=\psi(s)$, where $\psi:\left(\dot{\mathbb{R}}^{+}\right)^{n} \rightarrow\left(\dot{\mathbb{R}}^{+}\right)^{n}$ is a $C^{1}$-map such that $\operatorname{det}\left(J(\psi(s)) \neq 0\right.$ for all $s \in\left(\dot{\mathbb{R}}^{+}\right)^{n}$. Furthermore, (A) is satisfied if and only if the same assumption (A) holds after making a change of variable $t=\psi(s)$.

\section{Some Basic EXtreme Values}

In this section, using $\lambda(u), \Lambda(u)$ we introduce some extreme values of the Nehari manifold method that we believe are common for most problems.

Introduce

$$
\begin{aligned}
& \lambda_{\text {min }}=\inf _{u \in \mathcal{W}} \lambda(u) \equiv \inf _{u \in \mathcal{W}} \inf _{t \in\left(\mathbb{R}^{+}\right)^{n}} r(t \cdot u), \\
& \lambda_{\max }=\sup _{u \in \mathcal{W}} \Lambda(u) \equiv \sup _{u \in \mathcal{W}} \sup _{t \in\left(\mathbb{R}^{+}\right)^{n}} r(t \cdot u) .
\end{aligned}
$$

Similarly, define $\lambda_{\min }^{s c}, \lambda_{\max }^{s c}$ for the scalar fibered NG-Rayleigh's quotient $r(t u)$ instead of $r(t \cdot u)$.

\section{Lemma 4.1.}

(1) If $\lambda_{\min }>-\infty\left(\lambda_{\max }<+\infty\right)$, then $\mathcal{N}_{\lambda}=\emptyset$ for any $\lambda<\lambda_{\min }\left(\lambda>\lambda_{\max }\right)$.

(2) $\mathcal{N}_{\lambda}^{s c} \neq \emptyset$ for any $\lambda \in\left(\lambda_{\text {min }}^{s c}, \lambda_{\text {max }}^{s c}\right)$.

Proof. (1) follows immediately, since for $u \in \mathcal{N}_{\lambda}$, we have $r(u)=\lambda$. (2) holds, because by Corollary 3.1. $u \in \mathcal{N}_{\lambda}^{s c}$ if and only if $r(u)=\lambda$.

Note that since any solution of (2.1) belongs $\mathcal{N}_{\lambda}$, the values $\lambda_{\min }, \lambda_{\max }$ set bound the existence of any solution of (2.1), that is for all $\lambda\left\langle\lambda_{\min }\right.$ and $\left.\lambda\right\rangle \lambda_{\max }$ (provided $\lambda_{\min }>-\infty, \lambda_{\max }<+\infty$ ) equation (2.1) has no solutions in $W$.

The next assumption is technical. It specifies the shape of $r(t \cdot u)$ for which we prove our main results. 
(S) For any $u \in \mathcal{W}$ and $t \in\left(\dot{\mathbb{R}}^{+}\right)^{n}$, s.t. $t \cdot u \in \mathcal{N}_{r(t \cdot u)}$, the condition $\nabla_{t} r(t \cdot u)=0_{n}$ implies that the function $r(t \cdot u)$ attains its global minimum or/and maximum at the point $t$ in $\left(\dot{\mathbb{R}}^{+}\right)^{n}$.

Remark 4.2. In other words, condition $(\mathbf{S})$ means that for every $u \in \mathcal{W}$ one of the following holds: (i) $r(t \cdot u)$ has no critical point $t \in\left(\dot{\mathbb{R}}^{+}\right)^{n}$ such that $t \cdot u \in \mathcal{N}_{r(t \cdot u)}$; (ii) $r(t \cdot u)$ has only one critical point $t_{u} \in\left(\dot{\mathbb{R}}^{+}\right)^{n}$ such that $t_{u} u \in \mathcal{N}_{r(t \cdot u)}$, moreover, $r(t \cdot u)$ attains its global minimum or maximum at $t_{u}$ in $\left(\dot{\mathbb{R}}^{+}\right)^{n} ;($ iii $) \nabla_{t} r(t \cdot u) \equiv 0_{n}$ for all $t \in\left(\dot{\mathbb{R}}^{+}\right)^{n}$ that is $r(t \cdot u)$ is a constant in $\left(\dot{\mathbb{R}}^{+}\right)^{n}$ and attains its global minimum and maximum at any $t \in\left(\mathbb{R}^{+}\right)^{n}$.

Remark 4.3. In the case of scalar $N M M$, since $t u \in \mathcal{N}_{r(t u)}^{s c}$ for any $u \in \mathcal{W}, t>0$, condition (S) can be written as follows: for every $u \in \mathcal{W}$ one of the following holds: (i) $r(t u)$ has no critical points in $\dot{\mathbb{R}}^{+}$; (ii) $r(t u)$ has only one critical point $t \in \dot{\mathbb{R}}^{+}$; (iii) $\nabla_{t} r(t u) \equiv 0$ for all $t \in \dot{\mathbb{R}}^{+}$.

Remark 4.4. There is a special case where $r(t u)$ satisfies the following shape condition: (s00) has no critical point in $\dot{\mathbb{R}}^{+}$for any $u \in \mathcal{W}$. This implies, in particular, that for each $u \in \dot{W}$ the function $\alpha_{u}(t):=\Phi_{\lambda}(t u)$ has only one extreme value in $\dot{\mathbb{R}}^{+}$. The latter condition, in turn, means that in fact we are dealing with nonparametric Nehari manifolds. Observe, as per Poincare's program on the hierarchy of degeneracy by codimension (see [1), first we have to study the generic cases, that is nonparametric Nehari manifolds. However, the present work does not focus on this special case. For further insight into this problem, we refer the reader to [40].

Introduce

$$
\begin{aligned}
& \lambda_{\text {min }}^{*}=\sup _{u \in \mathcal{W}} \lambda(u) \equiv \sup _{u \in \mathcal{W}} \inf _{t \in\left(\mathbb{R}^{+}\right)^{n}} r(t \cdot u), \\
& \lambda_{\text {max }}^{*}=\inf _{u \in \mathcal{W}} \Lambda(u) \equiv \inf _{u \in \mathcal{W}} \sup _{t \in\left(\mathbb{R}^{+}\right)^{n}} r(t \cdot u) .
\end{aligned}
$$

Similarly, define $\lambda_{\min }^{s c, *}, \lambda_{\max }^{s c, *}$ for the scalar fibered NG-Rayleigh's quotient.

Evidently $\lambda_{\min } \leq \lambda_{\min }^{*}$ and $\lambda_{\max }^{*} \leq \lambda_{\max }$. Consequently, in the case of scalar $\mathrm{NMM}$, if $\lambda_{\text {min }}^{s c, *}<\lambda_{\text {max }}^{s c, *}$, then for any $\lambda \in\left(\lambda_{\text {min }}^{s c, *}, \lambda_{\text {max }}^{s c, *}\right)$ the Nehari manifold $\mathcal{N}_{\lambda}^{s c}$ is nonempty.

Lemma 4.5. Suppose (A), (S) are satisfied and $\lambda_{\min }^{*}<\lambda_{\max }^{*}$. Then for any $u \in \mathcal{N}_{\lambda}$, det $J\left(\nabla_{u} \Phi_{\lambda}(u)(u)\right) \neq 0$ provided $\lambda \in\left(\lambda_{\text {min }}^{*}, \lambda_{\text {max }}^{*}\right)$.

Proof. Let $\lambda \in\left(\lambda_{\text {min }}^{*}, \lambda_{\text {max }}^{*}\right)$ and $u \in \mathcal{N}_{\lambda}$. Suppose, contrary to our claim, that $\operatorname{det} J\left(\nabla_{u} \Phi_{\lambda}(u)(u)\right)=0$. Note that $\lambda=r(u)$, since $u \in \mathcal{N}_{\lambda}$. Therefore by (A), the point $t_{u}=1_{n}$ is an extremal for the function $r(t u)$. Then (S) yields that at the point $t_{u}=1_{n}$ the function $r(t \cdot u)$ attains its global minimum or/and maximum. Assume for instance that this is a global minimum point. Since $\lambda>\lambda_{\min }^{*}$, by (4.3) one has

$$
\min _{t \in\left(\mathbb{R}^{+}\right)^{n}} r(t \cdot u)=\lambda>\inf _{t \in\left(\mathbb{R}^{+}\right)^{n}} r(t \cdot u) .
$$

Thus we get a contradiction. The same conclusion can be drawn when $t_{u}=1_{n}$ is the point of global maximum of $r(t \cdot u)$.

Theorem 4.6. Suppose (A), (S) are satisfied and $\lambda_{\min }^{*}<\lambda_{\max }^{*}$. Assume $\lambda \in$ $\left(\lambda_{\text {min }}^{*}, \lambda_{\text {max }}^{*}\right)$. Then any solution $u_{\lambda}$ of (2.3) satisfies equation (2.1). 
Proof. Let $\lambda \in\left(\lambda_{\min }^{*}, \lambda_{\max }^{*}\right)$ and $u_{\lambda}$ be a solution of (2.3). Then Lemma 4.5 yields that $u_{\lambda}$ satisfies condition (2.5) and therefore by Lemma 2.1 the theorem follows.

Consider the following particular case of (S):

(S0): For any $u \in \dot{W}$ one of the following holds:

(i): $r(t \cdot u)$ has no critical point $t \in\left(\dot{\mathbb{R}}^{+}\right)^{n}$ such that $t u \in \mathcal{N}_{r(t \cdot u)}$;

(ii): $\nabla_{t} r(t \cdot u) \equiv 0_{n}$ for all $t \in\left(\dot{\mathbb{R}}^{+}\right)^{n}$.

Remark 4.7. Taking into account Remark 3.4 we see that, in the case of scalar NMM, condition (S0) implies (A).

Theorem 4.8. Suppose (A), (S0) hold and $-\infty \leq \lambda_{\min }<\lambda_{\max }^{*}$. Assume $\lambda \in$ $\left(\lambda_{\min }, \lambda_{\max }^{*}\right)$. Then any solution $u_{\lambda}$ of (2.3) satisfies equation (2.1).

Proof. As above, to prove the assertion it is sufficient to show that condition (2.5) is satisfied. Assume $\operatorname{det} J\left(\nabla_{u} \Phi_{\lambda}(u)(u)\right)=0$. By (A), $t_{u_{\lambda}}=1_{n}$ is the extremal point for the function $r(t \cdot u)$ and consequently $\left.\nabla_{t} r(t \cdot u)\right|_{t=1_{n}}=0_{n}$. Since $u_{\lambda} \in \mathcal{N}_{\lambda} \equiv \mathcal{N}_{r(u)}$, (S0) entails that the function $r\left(t \cdot u_{\lambda}\right)$ identically equals to the constant $\lambda$ in $\left(\mathbb{R}^{+}\right)^{n}$ and attains its global minimum and maximum at any point $t \in\left(\mathbb{R}^{+}\right)^{n}$. However, the assumption $\lambda<\lambda_{\text {max }}^{*}$ yields that $\lambda<\sup _{t \in\left(\mathbb{R}^{+}\right)^{n}} r\left(t \cdot u_{\lambda}\right)=$ $\max _{t \in\left(\mathbb{R}^{+}\right)^{n}} r\left(t \cdot u_{\lambda}\right) \equiv r\left(u_{\lambda}\right)=\lambda$. Thus we get a contradiction.

Clearly, this proof also contains

Lemma 4.9. Suppose (A), (S0) hold and $\lambda_{\min }<\lambda_{\text {max }}^{*}$. Then for any $u \in \mathcal{N}_{\lambda}$, $\operatorname{det} J\left(\nabla_{u} \Phi_{\lambda}(u)(u)\right) \neq 0$ provided $\lambda \in\left(\lambda_{\min }, \lambda_{\text {max }}^{*}\right)$.

Remark 4.10. In the present work we do not deal with the applicability of Nehari manifolds method at its extremal points like $\lambda_{\max }^{*}, \lambda_{\max }$ or $\lambda_{\min }$. This is a subject of another work.

Arguing as in the proof of Proposition 2.5] it can be proved

Corollary 4.11. Suppose (A), (S) ((S0)) hold and $\lambda_{\min }^{*}<\lambda_{\max }^{*}\left(\lambda_{\min }<\lambda_{\max }^{*}\right)$. Then $\mathcal{N}_{\lambda}$ is a $C^{1}$-manifold provided $\lambda \in\left(\lambda_{\text {min }}^{*}, \lambda_{\text {max }}^{*}\right)\left(\lambda \in\left(\lambda_{\text {min }}, \lambda_{\text {max }}^{*}\right)\right)$.

Let us stress that

$$
\Lambda^{s c}(u):=\sup _{t \in \mathbb{R}^{+}} r(t u) \leq \sup _{t \in\left(\mathbb{R}^{+}\right)^{n}} r(t \cdot u)=: \Lambda(u),
$$

and therefore,

$$
\lambda_{\max }^{s c, *}:=\inf _{u \in \mathcal{W}} \Lambda^{s c}(u) \leq \inf _{u \in \mathcal{W}} \Lambda(u)=: \lambda_{\max }^{*}
$$

Similarly,

$$
\lambda_{\max }^{s c} \leq \lambda_{\max }, \lambda_{\min } \leq \lambda_{\min }^{s c}, \lambda_{\min }^{*} \leq \lambda_{\min }^{s c, *} .
$$

Remark 4.12. It is obviously that the assumptions (S), (S0) and the definition of the extremal values $\lambda_{\min }, \lambda_{\max }, \lambda_{\min }^{*}, \lambda_{\max }^{*}$ etc. do not depend on of the change of variables as to Remark 3.5 . 


\section{EXtreme VAlues OF NMM IN EXPLiCIT VARIATIONAL FORMS}

In this section, applying the above theory we present some examples where the extreme values of NMM can be expressed in an explicit variational form.

\section{Example 1. (Problem with indefinite nonlinearity)}

Consider the following boundary value problem with indefinite nonlinearity

$$
\left\{\begin{array}{l}
-\Delta_{p} u=\lambda|u|^{p-2} u+f(x)|u|^{\gamma-2} u \text { in } \Omega \\
u=0 \text { on } \partial \Omega
\end{array}\right.
$$

where $\Omega$ is a bounded domain in $\mathbb{R}^{N}$ with smooth boundary; $\lambda \in \mathbb{R} ; \Delta_{p}(\cdot):=$ $\operatorname{div}\left(|\nabla(\cdot)|^{p-2} \nabla(\cdot)\right)$ is the $p$-Laplacian; $f \in L^{\infty}(\Omega)$ and we assume

$$
1<p<\gamma \leq p^{*}, \text { where } p^{*}= \begin{cases}\frac{p N}{N-p} & \text { if } p<N \\ +\infty & \text { if } p \geq N\end{cases}
$$

Subsequently, $W:=W_{0}^{1, p}(\Omega)$ denotes the standard Sobolev space with the norm

$$
\|u\|_{1}=\left(\int_{\Omega}|\nabla u|^{p} d x\right)^{1 / p}
$$

By a solution of (5.1) we shall mean a weak solution $u \in W:=W_{0}^{1, p}(\Omega)$. In what follows, $\lambda_{1}:=\lambda_{1, p}, \phi_{1}:=\phi_{1, p}$ denote the first eigenpair of the operator $-\Delta_{p}$ in $\Omega$ with zero boundary conditions. It is known that the eigenvalue $\lambda_{1}$ is positive, simple and isolated, the corresponding eigenfunction $\phi_{1}$ is positive and it can be normalized so that $\left\|\phi_{1}\right\|_{1}=1$ [5, 15, 32. In the case when $f$ may change the sign in $\Omega$, the nonlinearity in right hand side of (5.1) is called indefinite in sign (cf. [4, 6] ).

The boundary value problems with indefinite nonlinearity have been studied in a number of papers (see e.g. [4, 6, 10, 13, 20, 25, 18, 35]). An important role in these studies plays the following extremal value

$$
\lambda^{*}=\inf \left\{\frac{\int_{\Omega}|\nabla u|^{p} d x}{\int_{\Omega}|u|^{p} d x}: \quad \int_{\Omega} f(x)|u|^{\gamma} d x \geq 0, u \in W \backslash 0\right\},
$$

which, as far as we know, was first found by Ouyang [35].

Note that

$$
\lambda^{*}>\lambda_{1} \text { if and only if } \int_{\Omega} f(x)\left|\phi_{1}\right|^{\gamma} d x<0 .
$$

Let us show that (5.3) can be obtained by applying the method of NG-Rayleigh's quotient.

Lemma 5.1. $\lambda^{*}$ is the extreme value of NMM, namely it coincides with (4.4), i.e. $\lambda^{*}=\lambda_{\max }^{*}$.

Proof. Let

$$
\Phi_{\lambda}(u)=\frac{1}{p} \int|\nabla u|^{p} d x-\lambda \frac{1}{p} \int|u|^{p} d x-\frac{1}{\gamma} \int f|u|^{\gamma} d x, u \in W .
$$

Evidently, $\Phi_{\lambda} \in C^{1}(W, \mathbb{R}), \partial_{t} \tilde{\Phi}_{\lambda} \in C^{1}\left(\mathbb{R}^{+} \times W, \mathbb{R}\right)$. Note that $\Phi_{\lambda} \notin C^{2}(W \backslash 0, \mathbb{R})$ if $1<p<2$. 
Consider the corresponding Nehari manifold minimization problem

$$
\left\{\begin{array}{l}
\frac{1}{p} \int|\nabla u|^{p} d x-\lambda \frac{1}{p} \int|u|^{q} d x-\frac{1}{\gamma} \int f|u|^{\gamma} d x \rightarrow \min \\
\int|\nabla u|^{p} d x-\lambda \int|u|^{p} d x-\int f|u|^{\gamma} d x=0, \quad u \in W \backslash 0 .
\end{array}\right.
$$

Consider the NG-Rayleigh's quotient corresponding to (5.1)

$$
r(u)=\frac{\int_{\Omega}|\nabla u|^{p} d x-\int_{\Omega} f(x)|u|^{\gamma} d x}{\int_{\Omega}|u|^{p} d x}, u \in W \backslash 0
$$

Let $u \in W \backslash 0$. Observe, for $t>0$

$$
r(t u)=\frac{\int_{\Omega}|\nabla u|^{p} d x}{\int_{\Omega}|u|^{p} d x}-t^{\gamma-p} \frac{\int_{\Omega} f(x)|u|^{\gamma} d x}{\int_{\Omega}|u|^{p} d x} .
$$

Hence,

$$
\Lambda(u)=\sup _{t>0} r(t u)= \begin{cases}\frac{\int_{\Omega}|\nabla u|^{p} d x}{\int_{\Omega}|u|^{p} d x}, & \text { if } \int_{\Omega} f(x)|u|^{\gamma} d x \geq 0 \\ +\infty, & \text { if } \quad \int_{\Omega} f(x)|u|^{\gamma} d x<0\end{cases}
$$

and

$$
\lambda(u)=\inf _{t>0} r(t u)=\left\{\begin{array}{l}
-\infty, \text { if } \int_{\Omega} f(x)|u|^{\gamma} d x>0, \\
\frac{\int_{\Omega}|\nabla u|^{p} d x}{\int_{\Omega}|u|^{p} d x}, \quad \text { if } \int_{\Omega} f(x)|u|^{\gamma} d x \leq 0 .
\end{array}\right.
$$

Consequently, we obtain for extreme values (4.3), (4.4) the following explicit variational forms

$$
\begin{aligned}
& \lambda_{\text {min }}^{*}=\sup \left\{\frac{\int_{\Omega}|\nabla u|^{p} d x}{\int_{\Omega}|u|^{p} d x}: \quad \int_{\Omega} f(x)|u|^{\gamma} d x \leq 0, u \in W \backslash 0\right\}, \\
& \lambda_{\text {max }}^{*}=\inf \left\{\frac{\int_{\Omega}|\nabla u|^{p} d x}{\int_{\Omega}|u|^{p} d x}: \quad \int_{\Omega} f(x)|u|^{\gamma} d x \geq 0, u \in W \backslash 0\right\} .
\end{aligned}
$$

Thus we see that (5.8) coinsides with (5.3).

It is easily to see that $r(t u)$ has only extremal point at $t=0$ or in the case $\int_{\Omega} f(x)|u|^{\gamma} d x=0, r(t u) \equiv \frac{\int_{\Omega}|\nabla u|^{p} d x}{\int_{\Omega}|u|^{p} d x}$ for all $t \geq 0$. Thus condition (S0) is satisfied and consequently (A) holds (see Remark 4.7).

Observe, $\lambda_{\text {min }}^{*}=-\infty$ if $\int_{\Omega} f(x)|u|^{\gamma} d x>0$ for all $u \in W \backslash 0$, and $\lambda_{\text {min }}^{*}=+\infty$ if the set $\{x \in \Omega: f(x) \leq 0\}$ contains an open domain up to a subset of Lebesgue measure zero. Note that when $\int_{\Omega} f(x)|u|^{\gamma} d x>0$ for all $u \in W \backslash 0$ we have $\lambda_{\max }^{*}=\lambda_{1}$. Thus, we have a strong inequality $\lambda_{\min }^{*}<\lambda_{\max }^{*}=\lambda_{1}$ only if $f>0$ a.e. in $\Omega$, and therefore only in this case we may apply Theorem 4.6 .

However, for another extremal value of NMM $\lambda_{\min }:=\inf _{u \in W \backslash 0} \lambda(u)$ we have $\lambda_{\text {min }}=-\infty$ if $\int_{\Omega} f(x)|u|^{\gamma} d x>0$ for some $u \in W \backslash 0$, and $\lambda_{\text {min }}=\lambda_{1}, \lambda_{\text {max }}^{*}=+\infty$ if $\int_{\Omega} f(x)|u|^{\gamma} d x \leq 0$ for all $u \in W \backslash 0$. Thus we always have $\lambda_{\min }<\lambda_{\max }^{*}$. Thus Theorem 4.8 yields

Lemma 5.2. Assume $1<p<\gamma \leq p^{*}$ and $\lambda \in\left(\lambda_{\min }, \lambda_{\max }^{*}\right)$. Then any solution $u_{\lambda}$ of (5.5) satisfies equation (5.1). 
The proof of the existence of the solution of Nehari manifold minimization problem (5.5) when $\lambda \in\left(\lambda_{\min }, \lambda_{\max }^{*}\right)$ can be found in 4, 35) (for $\gamma<p^{*}, p=2$ ), in [13, 23, 25, 26] ( for $1<p<+\infty$ ) and in [18, (for $\gamma \leq p^{*}$ ). Furthermore, it can be proved that if $\lambda \in\left(\lambda_{1}, \lambda_{\max }^{*}\right)$ then (5.5) has two nonnegative solutions (see e.g. [4, 35, 23]).

\section{Example 2. (Problem with convex-concave nonlinearity)}

Consider the following problem with convex-concave nonlinearity

$$
\left\{\begin{array}{l}
-\Delta_{p} u=\lambda|u|^{q-2} u+f(x)|u|^{\gamma-2} u, x \in \Omega, \\
\left.u\right|_{\partial \Omega}=0,
\end{array}\right.
$$

where $\Omega$ is a bounded domain in $\mathbb{R}^{N}, N \geq 1$, with smooth boundary $\partial \Omega, \Delta_{p}$ is the p-Laplacian and we assume that $1<q<p<\gamma \leq p^{*}$.

We always suppose that $f(x) \geq 0$ on $\Omega$ and $f \in L^{d}(\Omega)$, where $d>p^{*} /\left(p^{*}-\gamma\right)$ if $p<N$ and $\gamma<p^{*} ; d=+\infty$ if $p<N$ and $\gamma=p^{*} ; d>1$ if $p \geq N$. By a solution of (5.2) we shall mean a weak solution $u \in W_{0}^{1, p}(\Omega)$.

The investigation of the problems with convex-concave type nonlinearity similar to (5.9) can be found in various papers (see e.g. [2, 3, 24, 11, 31]). In particular, in 24] has been found using the so-called spectral analysis by the fibering method the following extremal value of NMM

$$
\lambda^{*}=\frac{\gamma-p}{p-q}\left(\frac{p-q}{\gamma-q}\right)^{\frac{\gamma-q}{\gamma-p}} \inf _{u \in W \backslash 0}\left(\frac{\left(\int|\nabla u|^{p} d x\right)^{\frac{\gamma-q}{\gamma-p}}}{\left(\int|u|^{q} d x\right)\left(\int f|u|^{\gamma} d x\right)^{\frac{p-q}{\gamma-p}}}\right) .
$$

Let us show that (5.10) can be obtained also by applying the method of NGRayleigh's quotient.

Lemma 5.3. $\lambda^{*}$ is the extreme value of NMM, namely it coincides with (4.4), i.e. $\lambda^{*}=\lambda_{\max }^{*}$.

Proof. Let

$$
\Phi_{\lambda}(u)=\frac{1}{p} \int|\nabla u|^{p} d x-\lambda \frac{1}{q} \int|u|^{q} d x-\frac{1}{\gamma} \int|u|^{\gamma} d x .
$$

The Nehari manifold minimization problem for (5.9) is given by

$$
\left\{\begin{array}{l}
\frac{1}{p} \int|\nabla u|^{p} d x-\lambda \frac{1}{q} \int|u|^{q} d x-\frac{1}{\gamma} \int|u|^{\gamma} d x \rightarrow \min \\
\int|\nabla u|^{p} d x-\lambda \int|u|^{q} d x-\int f|u|^{\gamma} d x=0, \quad u \in \dot{W},
\end{array}\right.
$$

and the corresponding NG-Rayleigh's quotient is

$$
r(u)=\frac{\int|\nabla u|^{p} d x-\int|u|^{\gamma} d x}{\int f|u|^{q} d x} .
$$

Observe for $u \in W \backslash 0, t>0$

$$
r(t u)=\frac{t^{p-q} \int|\nabla u|^{p} d x-t^{\gamma-q} \int f|u|^{\gamma} d x}{\int|u|^{q} d x} .
$$

Compute

$$
\frac{\partial}{\partial t} r(t u)=\frac{(p-q) t^{p-q-1} \int|\nabla u|^{p} d x-(\gamma-q) t^{\gamma-q-1} \int f|u|^{\gamma} d x}{\int|u|^{q} d x}
$$


Hence, $\frac{\partial}{\partial t} r(t u)=0$ if and only if

$$
(p-q) t^{p-q-1} \int|\nabla u|^{p} d x-(\gamma-q) t^{\gamma-q-1} \int f|u|^{\gamma} d x=0 .
$$

The only solution of this equation is

$$
t_{u, \max }=\left(\frac{(p-q) \int|\nabla u|^{p} d x}{(\gamma-q) \int f|u|^{\gamma} d x}\right)^{\frac{1}{\gamma-p}} .
$$

The substituting $t_{u, \max }$ into $r(t u)$ yields

$$
\Lambda(u)=r\left(t_{u, \max } u\right)=\frac{\gamma-p}{p-q}\left(\frac{p-q}{\gamma-q}\right)^{\frac{\gamma-q}{\gamma-p}}\left(\frac{\left(\int|\nabla u|^{p} d x\right)^{\frac{\gamma-q}{\gamma-p}}}{\left(\int|u|^{q} d x\right)\left(\int|u|^{\gamma} d x\right)^{\frac{p-q}{\gamma-p}}}\right)
$$

Thus, indeed $\lambda_{\text {max }}^{*}=\inf _{u \in \dot{W}} \Lambda(u)$ coincides with (5.10).

Obviously, conditions (A) and (S) hold (see Remark 3.4). It is not hard to show using Sobolev's imbedding theorem (see 24]) that $\lambda_{\max }^{*}>0$. Notice

$$
\lambda(u)=\inf _{t>0} r(t u)=-\infty, \quad \forall u \in W \backslash 0,
$$

and therefore $\lambda_{\min }=\lambda_{\min }^{*}=-\infty$. Thus Theorem 4.6 yields

Lemma 5.4. Assume (5.2) holds. Then $\lambda_{\max }^{*}>0$ and for $\lambda \in\left(-\infty, \lambda_{\text {max }}^{*}\right)$ any solution $u_{\lambda}$ of (5.12) satisfies equation (5.9).

For the existence of the solution of (5.12) when $\lambda \in\left(-\infty, \lambda_{\max }^{*}\right)$ we refer the reader to [2, 3, 24, 11] where the existence of two distinct nonnegative solutions (5.9) for $\lambda \in\left(0, \lambda_{\max }^{*}\right)$ is proven as well. However, the proof of these assertions can be found also below in Section 7, where we prove similar results for general convex-concave problems which contain (5.12) as a particular case.

\section{Example 3. (System of equations with convex-concave nonlinearity)}

Consider system of equations with convex-concave nonlinearity

$$
\begin{cases}-\Delta_{p} u=\lambda|u|^{q-2} u+\alpha f(x)|u|^{\alpha-2} u|v|^{\beta}, & x \in \Omega, \\ -\Delta_{p} v=\lambda|v|^{q-2} v+\beta f(x)|u|^{\alpha}|v|^{\beta-2} v, & x \in \Omega, \\ \left.u\right|_{\partial \Omega}=0,\left.v\right|_{\partial \Omega}=0, & \end{cases}
$$

where $\Omega \subset \mathbb{R}^{N}, N \geq 1$ is a bounded domain with $C^{1}$-boundary $\partial \Omega, \lambda, \mu \in \mathbb{R}$, $1<q<p<\alpha+\beta \leq p^{*}$. We suppose $f \in L^{\infty}(\Omega)$ and $f \geq 0$ in $\Omega$. By a solution of (5.1) we shall mean a weak solution $(u, v) \in W:=W_{0}^{1, p}(\Omega) \times W_{0}^{1, q}(\Omega)$.

The problem has a variational form with the Euler-Lagrange functional given by

$\Phi_{\lambda}(u, v)=\frac{1}{p} \int\left(|\nabla u|^{p}+|\nabla v|^{p}\right) d x+\lambda \frac{1}{q} \int\left(|u|^{q}+\int|v|^{q}\right) d x-\int f(x)|u|^{\alpha}|v|^{\beta} d x$,

for $(u, v) \in W$. Evidentely, $\Phi_{\lambda} \in C^{1}(\dot{W}, \mathbb{R}), \nabla_{t} \tilde{\Phi}_{\lambda} \in C^{1}\left(\left(\dot{\mathbb{R}}^{+}\right)^{n} \times \dot{W}, \mathbb{R}^{n}\right)$. However, $\Phi_{\lambda} \notin C^{2}(\dot{W}, \mathbb{R})$ if $1<p<2$ or/and $1<q<2$.

The corresponding NG-Rayleigh's quotient is defined as follows

$$
r(u, v)=\frac{\int|\nabla u|^{p} d x+\int|\nabla v|^{p} d x-(\alpha+\beta) \int f(x)|u|^{\alpha}|v|^{\beta} d x}{\int|u|^{q} d x+\int|v|^{q} d x} .
$$

For (5.18) we may apply two methods: the vector NMM (2.3) or the scalar NMM (2.7). Let us consider both of them. 


\section{Scalar Nehari manifold method.}

The scalar Nehari manifold minimization problem corresponding to (5.18) is defined as follows

$$
\left\{\begin{array}{l}
\Phi_{\lambda}(u, v) \rightarrow \min \\
\int\left(|\nabla u|^{p}+|\nabla v|^{p}\right) d x+\lambda \int\left(|u|^{q}+\int|v|^{q}\right) d x- \\
(u, v) \in \dot{W} .
\end{array}\right.
$$

Consider

$$
r(t(u, v))=\frac{t^{p-q}\left(\int\left(|\nabla u|^{p}+|\nabla v|^{p}\right) d x-(\alpha+\beta) t^{\alpha+\beta-q} \int f(x)|u|^{\alpha}|v|^{\beta} d x\right.}{\int\left(|u|^{q}+\int|v|^{q}\right) d x},
$$

where $t>0,(u, v) \in W \backslash 0_{2}$. It is easily seen that in this case we may apply the same analysis as it has been done above for (5.12), (5.14). In this way, we introduce

$$
\Lambda^{s c}(u, v)=C_{p, q, \alpha, \beta}\left(\frac{\left(\int\left(|\nabla u|^{p}+|\nabla v|^{p}\right) d x\right)^{\frac{\alpha+\beta-q}{\alpha+\beta-p}}}{\left(\int\left(|u|^{q}+\int|v|^{q}\right) d x\right)\left((\alpha+\beta) \int f(x)|u|^{\alpha}|v|^{\beta} d x\right)^{\frac{p-q}{\alpha+\beta-p}}}\right)
$$

where $C_{p, q, \alpha, \beta}$ is a constant which does not depend on $(u, v) \in \dot{W}$. Thus for the extreme value (4.4) express as the following explicit variational form

$$
\lambda_{\max }^{s c, *}=\inf _{(u, v) \in W \backslash 0_{2}} \Lambda^{s c}(u, v) .
$$

As above, it is not hard to show using Sobolev's imbedding theorem that $\lambda_{\text {max }}^{s c, *}>0$. Thus Theorem 4.6 yields

Lemma 5.5. Assume $1<q<p<\alpha+\beta \leq p^{*}$. Then $\lambda_{\max }^{s c, *}>0$ and for $\lambda \in$ $\left(-\infty, \lambda_{\max }^{s c, *}\right)$ any solution $u_{\lambda}$ of (5.19) satisfies equation (5.18).

The existence of the solution for $\lambda \in\left(-\infty, \lambda_{\max }^{s c, *}\right)$ (and even multiple solutions for $\left.\lambda \in\left(0, \lambda_{\max }^{s c, *}\right)\right)$ of (5.19) can be obtained using Theorems 3.1 and 3.2 from [11]. Note that in [11] the existence of solutions of (5.18) is proven only locally by $\lambda$, namely, for $\lambda \in(0, \delta)$ with some sufficiently small $\delta$.

Vector Nehari manifold method.

Now consider the corresponding vector Nehari manifold minimization problem

$$
\left\{\begin{array}{l}
\Phi_{\lambda}(u, v) \rightarrow \min \\
\int|\nabla u|^{p} d x-\lambda \int|u|^{q} d x-\alpha \int f(x)|u|^{\alpha}|v|^{\beta} d x=0 \\
\int|\nabla v|^{p} d x-\lambda \int|v|^{q} d x-\beta \int f(x)|u|^{\alpha}|v|^{\beta} d x=0 \\
(u, v) \in \dot{W} .
\end{array}\right.
$$

Let us find the extremal value (4.4) corresponding to (5.20). Taking into account Remark 4.12 we have

$$
\Lambda(u, v)=\sup _{(t, s)>0} r(t u, t s v)=\frac{t^{p} \int\left(|\nabla u|^{p}+s^{p}|\nabla v|^{p}\right) d x-t^{\alpha+\beta} s^{\beta} \int f(x)|u|^{\alpha}|v|^{\beta} d x}{t^{q} \int\left(|u|^{q}+s^{q} \int|v|^{q}\right) d x} .
$$

Similar to (5.17) we see that for every $s>0$ there holds

$$
\sup _{t>0} r(t u, t s v)=C_{p, q, \alpha, \beta}\left(\frac{\left(\int\left(|\nabla u|^{p}+s^{p}|\nabla v|^{p}\right) d x\right)^{\frac{\alpha+\beta-q}{\gamma-p}}}{\left(\int\left(|u|^{q}+s^{q} \int|v|^{q}\right) d x\right)\left(s^{\beta}(\alpha+\beta) \int f(x)|u|^{\alpha}|v|^{\beta} d x\right)^{\frac{p-q}{\alpha+\beta-p}}}\right) .
$$


Using this fact it is not hard to show that

$$
\lambda_{v, \text { max }}^{*}=\inf _{u \in W \backslash 0} \Lambda(u, v)=\inf _{u \in W \backslash 0} \Lambda(u, v)=\lambda_{\max }^{s c, *} .
$$

Thus, the extremal values for the vector (5.20) and the scalar (5.19) NMM are the same. The investigate of the existence of solution of (5.20) is left to the reader.

\section{Example 4. (System of equations with indefinite nonlinearity)}

Consider system of equations with indefinite nonlinearity

$$
\begin{cases}-\Delta_{p} u=\lambda|u|^{p-2} u+\alpha f(x)|u|^{\alpha-2} u|v|^{\beta}, & x \in \Omega, \\ -\Delta_{q} v=\lambda|v|^{q-2} v+\beta f(x)|u|^{\alpha}|v|^{\beta-2} v, & x \in \Omega, \\ \left.u\right|_{\partial \Omega}=0,\left.v\right|_{\partial \Omega}=0 & \end{cases}
$$

where $\Omega \subset \mathbb{R}^{N}, N \geq 1$ is a bounded domain with $C^{1}$-boundary $\partial \Omega, \lambda, \mu \in \mathbb{R}$, $1<p<+\infty, 1<q<+\infty$ and

$$
\alpha, \beta>0, \quad \frac{\alpha}{p}+\frac{\beta}{q}>1, \quad \frac{\alpha}{p^{*}}+\frac{\beta}{q^{*}}<1 .
$$

Here, as above, $p^{*}$ and $q^{*}$ are the standard critical Sobolev exponents. We suppose $f \in L^{\infty}(\Omega)$ and that the function $f$ may change the sign on $\Omega$, i.e. the problem (5.21) has indefinite nonlinearity. By a solution of (5.1) we shall mean a weak solution $(u, v) \in W:=W_{0}^{1, p}(\Omega) \times W_{0}^{1, q}(\Omega)$.

This system of equations has been studied, for instance, in [7, 8, 10. In particular, in 7] for (5.21) an extreme value of Nehari manifold method has been introduced. Here we obtain this value using the method of NG-Rayleigh's quotient.

Consider

$$
\Phi_{\lambda}(u, v)=\frac{1}{p} P_{\lambda}(u)+\frac{1}{q} Q_{\lambda}(v)-F(u, v), \quad(u, v) \in W,
$$

where

$$
\begin{aligned}
P_{\lambda}(u) & =\int|\nabla u|^{p} d x-\lambda \int|u|^{p} d x, \quad Q_{\lambda}(v)=\int|\nabla v|^{q} d x-\lambda \int|v|^{q} d x, \\
F(u, v) & =\int f(x)|u|^{\alpha}|v|^{\beta} d x .
\end{aligned}
$$

Then the corresponding Nehari manifold is defined as follows

$$
\mathcal{N}_{\lambda}:=\left\{(u, v) \in \dot{W}: \quad P_{\lambda}(u)-\alpha F(u, v)=0, \quad Q_{\lambda}(v)-\beta F(u, v)=0\right\},
$$

whereas the Nehari manifold minimization problem is

$$
\left\{\begin{array}{l}
\frac{1}{p} P_{\lambda}(u)+\frac{1}{q} Q_{\lambda}(v)-F(u, v) \rightarrow \min \\
P_{\lambda}(u)-\alpha F(u, v)=0 \\
Q_{\lambda}(u)-\beta F(u, v)=0 \\
(u, v) \in \dot{W}
\end{array}\right.
$$

Let us prove

Lemma 5.6. The extreme value (4.4) of (5.24) is expressed by the following explicit variational form

$$
\lambda_{\max }^{*}=\inf \left\{\max \left\{\frac{\int|\nabla u|^{p} d x}{\int|u|^{p} d x}, \frac{\int|\nabla v|^{q} d x}{\int|v|^{q} d x}\right\}: F(u, v) \geq 0,(u, v) \in \dot{W}\right\} .
$$


Proof. Consider NG-Rayleigh's quotient

$$
r(t u, s v)=\frac{t^{p} \int|\nabla u|^{p} d x+s^{q} \int|\nabla v|^{q} d x-t^{\alpha} s^{\beta}(\alpha+\beta) F(u, v)}{t^{p} \int|u|^{p} d x+s^{q} \int|v|^{q} d x} .
$$

We claim that

$$
\Lambda(u, v)=\sup _{(t, s)>0} r(t u, s v)=\left\{\begin{array}{l}
\max \left\{\frac{\int|\nabla u|^{p} d x}{\int|u|^{p} d x}, \frac{\int|\nabla v|^{q} d x}{\int|v|^{q} d x}\right\}, \text { if } F(u, v) \geq 0 \\
+\infty, \quad \text { if } F(u, v)<0
\end{array}\right.
$$

and

$$
\lambda(u, v)=\inf _{(t, s)>0} r(t u, s v)=\left\{\begin{array}{l}
-\infty, \text { if } F(u, v)>0, \\
\min \left\{\frac{\int|\nabla u|^{p} d x}{\int|u|^{p} d x}, \frac{\int|\nabla v|^{q} d x}{\int|v|^{q} d x}\right\}, \text { if } F(u, v) \leq 0 .
\end{array}\right.
$$

Let us show, as an example, (5.25). Assume $F(u, v)<0$. Then setting $t=\sigma^{q}$, $s=\sigma^{p}$ gives

$$
\frac{\int|\nabla u|^{p} d x+\int|\nabla v|^{q} d x-\sigma^{p q\left(\frac{\alpha}{p}+\frac{\beta}{q}-1\right)} F(u, v)}{\int|u|^{p} d x+\int|v|^{q} d x} \rightarrow+\infty
$$

for $\sigma \rightarrow+\infty$, since $\frac{\alpha}{p}+\frac{\beta}{q}>1$. Consider now the case $F(u, v) \geq 0$. Without loss of generality, we can suppose that $\frac{\int|\nabla u|^{p} d x}{\int|u|^{p} d x} \geq \frac{\int|\nabla v|^{q} d x}{\int|v|^{q} d x}$. This implies that

$$
\frac{\int|\nabla u|^{p} d x+\tau \int|\nabla v|^{q} d x}{\int|u|^{p} d x+\tau \int|v|^{q} d x} \leq \frac{\int|\nabla u|^{p} d x}{\int|u|^{p} d x}
$$

for any $\tau \geq 0$. Hence, since $F(u, v) \geq 0$,

$$
r(t u, s v)=\frac{\int|\nabla u|^{p} d x+s^{q} t^{-p} \int|\nabla v|^{q} d x-t^{\alpha-p} s^{\beta}(\alpha+\beta) F(u, v)}{\int|u|^{p} d x+s^{q} t^{-p} \int|v|^{q} d x} \leq \frac{\int|\nabla u|^{p} d x}{\int|u|^{p} d x} .
$$

for any $s \geq 0$ and $t>0$. Since for $s=0$ this inequity becomes equality, we get (5.25). Furthermore, we see that the supremum and infimum in (5.25), (5.26) are attained on the line $s=0$ or $t=0$.

Let us verify the assumption of Theorem 4.8. Find the corresponding Jacobian matrix

$$
\begin{aligned}
& J\left(\nabla_{(u, v)} \Phi_{\lambda}(u, v)(u, v)\right)= \\
& \left.\qquad \begin{array}{cc}
(p-1) P_{\lambda}(u)-\alpha(\alpha-1) F(u, v) & -\alpha \beta F(u, v) \\
-\alpha \beta F(u, v) & (q-1) Q_{\lambda}(v)-\beta(\beta-1) F(u, v)
\end{array}\right) .
\end{aligned}
$$

Then for $(u, v) \in \mathcal{N}_{\lambda}$ we have

$$
J\left(\nabla_{(u, v)} \Phi_{\lambda}(u, v)(u, v)\right)=\left(\begin{array}{cc}
\alpha(p-\alpha) F(u, v) & -\alpha \beta F(u, v) \\
-\alpha \beta F(u, v) & \beta(q-\beta) F(u, v)
\end{array}\right)
$$


and consequently

$$
\operatorname{det} J_{t}\left(\nabla_{(u, v)} \Phi_{\lambda}(u, v)(u, v)\right)=\alpha \beta(p q-p \beta-q \alpha) F^{2}(u, v) .
$$

Thus, since $\frac{\alpha}{p}+\frac{\beta}{q}>1$, if $(t u, s v) \in \mathcal{N}_{r(t u, s v)}$ and $\operatorname{det} J_{(t, s)}\left(\nabla_{(u, v)} \Phi_{\lambda}(t u, s v)(t u, s v)\right)=$ 0 for $t>0, s>0$, then $F(t u, s v)=0$ and hence $J_{(t, s)}\left(\nabla_{(u, v)} \Phi_{\lambda}(t u, s v)(t u, s v)\right) 1_{2}=$ $0_{2}$. Thus by (ii), Proposition 3.3 it follows that $r(t u, s v)$ satisfies condition (A).

Observe, for $(u, v) \in W \backslash 0_{2}, t>0, s>0$, we have

$$
\begin{aligned}
& \partial_{t} r(t u, s v)=\frac{1}{t\left(t^{p} \int|u|^{p} d x+s^{q} \int|v|^{q} d x\right)}\left(p P_{\lambda}(t u)-\alpha(\alpha+\beta) F(t u, s v)\right), \\
& \partial_{s} r(t u, s v)=\frac{1}{s\left(t^{p} \int|u|^{p} d x+s^{q} \int|v|^{q} d x\right)}\left(q Q_{\lambda}(s v)-\beta(\alpha+\beta) F(t u, s v)\right) .
\end{aligned}
$$

Thus, if we assume that $\partial_{t} r(t u, s v)=0, \partial_{s} r(t u, s v)=0$ and $(t u, s v) \in \mathcal{N}_{r(t u, s v)}$, then $P_{\lambda}(t u)=0, Q_{\lambda}(s v)=0$ and $F(t u, s v)=0$. Hence, since $t>0, s>0$, we have $P_{\lambda}(u)=0, Q_{\lambda}(v)=0$ and $F(u, v)=0$. This implies that $\partial_{t} r(t u, s v) \equiv 0$, $\partial_{s} r(t u, s v) \equiv 0$ for all $t>0, s>0$. Thus condition (S0) is satisfied.

As in the scalar case (5.1), it is readily seen that the extremal value $\lambda_{\min }^{*}=$ $\sup _{(u, v) \in \dot{W}} \lambda(u, v)$ is useless.

Introduce, $\lambda_{1}^{l}:=\min \left\{\lambda_{1, p}, \lambda_{1, q}\right\}, \lambda_{1}^{u}:=\max \left\{\lambda_{1, p}, \lambda_{1, q}\right\}$. Clearly,

$$
\begin{aligned}
& \lambda_{1}^{l}=\inf _{(u, v) \in W} \min \left\{\frac{\int|\nabla u|^{p} d x}{\int|u|^{p} d x}, \frac{\int|\nabla v|^{q} d x}{\int|v|^{q} d x}\right\}, \\
& \lambda_{1}^{u}=\inf _{(u, v) \in \dot{W}} \max \left\{\frac{\int|\nabla u|^{p} d x}{\int|u|^{p} d x}, \frac{\int|\nabla v|^{q} d x}{\int|v|^{q} d x}\right\} .
\end{aligned}
$$

Hence, $\lambda_{\max }^{*} \geq \lambda_{1}^{u} \geq \lambda_{1}^{l}$ and

$$
\lambda_{\max }^{*}>\lambda_{1}^{u} \text { iff } F\left(\phi_{1, p}, \phi_{1, q}\right)<0 .
$$

Note

$$
\lambda_{\text {min }}= \begin{cases}-\infty, & \text { if } \exists(u, v) \in \dot{W} \quad \text { s.t. } \quad F(u, v)>0, \\ \lambda_{1}^{l}, & \text { if } \forall(u, v) \in \dot{W}, \quad F(u, v) \leq 0 .\end{cases}
$$

Thus, since $\lambda_{\max }^{*}=+\infty$ if $F(u, v) \leq 0, \forall(u, v) \in \dot{W}$, we always have $\lambda_{\min }<\lambda_{\max }^{*}$.

Thus, all the assumptions of Theorem 4.8 are satisfied and therefore we have

Lemma 5.7. Assume(5.22) holds and $\lambda \in\left(\lambda_{\min }, \lambda_{\text {max }}^{*}\right)$. Then any solution $\left(u_{\lambda}, v_{\lambda}\right)$ of (5.24) satisfies system (5.21).

The existence of the solution of (5.24) for $\lambda \in\left(\lambda_{\min }, \lambda_{1}^{l}\right) \cup\left(\lambda_{1}^{u}, \lambda_{\max }^{*}\right)$ follows from [7, 8]. Note that if $\lambda_{1, p}=\lambda_{1, q}$ (for instance when $p=q$ ), then we have $\lambda_{1}^{l}=\lambda_{1}^{u}$. However, we conjecture that solutions of (5.21) can not be obtained by the Nehari manifold method if $\lambda \in\left[\lambda_{1}^{l}, \lambda_{1}^{u}\right]$. Let us give some comments for this.

Observe that the system of equations

$$
\left\{\begin{array}{l}
\frac{\partial}{\partial t} \Phi_{\lambda}(t u, s v) \equiv t^{p-1} P_{\lambda}(u)-\alpha t^{\alpha-1} s^{\beta} F(u, v)=0, \\
\frac{\partial}{\partial s} \Phi_{\lambda}(t u, s v) \equiv s^{q-1} Q_{\lambda}(v)-\beta t^{\alpha} s^{\beta-1} F(u, v)=0 .
\end{array}\right.
$$


has a solution $(t, s) \in \dot{\mathbb{R}}^{+} \times \dot{\mathbb{R}}^{+}$only if $(u, v)$ belongs to one of the following sets

$$
\begin{aligned}
& \mathcal{A}:=\left\{(u, v) \in \dot{W}: P_{\lambda}(u)>0, \quad Q_{\lambda}(v)>0, \quad F(u, v)>0\right\}, \\
& \mathcal{B}:=\left\{(u, v) \in \dot{W}: P_{\lambda}(u)<0, \quad Q_{\lambda}(v)<0, \quad F(u, v)<0\right\},
\end{aligned}
$$

Furthermore, the solution $t=t(u, v), s=s(u, v)$ of (5.28) is unique and given by

$$
\begin{aligned}
t^{p q d} & =\frac{\alpha^{\beta-q}}{\beta^{\beta}} \frac{\left|P_{\lambda}(u)\right|^{q-\beta}\left|Q_{\lambda}(v)\right|^{\beta}}{|F(u, v)|^{q}}, \\
s^{p q d} & =\frac{\beta^{\alpha-p}}{\alpha^{\alpha}} \frac{\left|P_{\lambda}(u)\right|^{\alpha}\left|Q_{\lambda}(v)\right|^{p-\alpha}}{|F(u, v)|^{p}},
\end{aligned}
$$

where

$$
d:=\frac{\alpha}{p}+\frac{\beta}{q}-1>0 .
$$

Substituting these roots into $\Phi_{\lambda}(t u, s v)$ we obtain the function

$$
\mathcal{J}_{\lambda}(u, v):=\Phi_{\lambda}(t(u, v) u, s(u, v) v)=C \frac{\left|P_{\lambda}(u)\right|^{\frac{\alpha}{p d}}\left|Q_{\lambda}(v)\right|^{\frac{\beta}{q d}}}{|F(u, v)|^{\frac{1}{d}}} \operatorname{sign}(F(u, v)),
$$

where

$$
C=\left(\frac{1}{\alpha^{\alpha q} \beta^{\beta p}}\right)^{\frac{1}{p q d}} d
$$

Note also that by the definition of $\lambda_{\max }^{*}$ we have: for $(u, v) \in W$

$$
\text { if } F(u, v) \geq 0, \text { then }\left\{\begin{array}{c}
\int|u|^{p} d x \leq \frac{1}{\lambda_{\text {max }}^{*}} \int|\nabla u|^{p} d x, \\
\text { or/and } \\
\int|v|^{q} d x \leq \frac{1}{\lambda_{\text {max }}^{*}} \int|\nabla v|^{q} d x
\end{array}\right.
$$

and

$$
\text { if }\left\{\begin{array}{c}
\int|u|^{p} d x>\frac{1}{\lambda_{\text {max }}^{*}} \int|\nabla u|^{p} d x, \\
\text { and } \\
\int|v|^{q} d x>\frac{1}{\lambda_{\text {max }}^{*}} \int|\nabla v|^{q} d x,
\end{array} \text { then } F(u, v)<0 .\right.
$$

Let us remark that $\mathcal{A} \neq \emptyset$ only if the following is satisfied

$$
\{x \in \Omega: f(x)>0\} \neq \emptyset \text { up to a subset of Lebesgue measure zero }
$$

Let $\lambda \in\left[\lambda_{1}^{l}, \lambda_{1}^{u}\right]$. Assume, for instance, that $\lambda_{1, q}=\lambda_{1}^{l}=\min \left\{\lambda_{1, p}, \lambda_{1, q}\right\}$ and $\lambda_{1, p}=\lambda_{1}^{u}=\max \left\{\lambda_{1, p}, \lambda_{1, q}\right\}$. Then $P_{\lambda}(u)>0$ for all $u \in \dot{W}_{0}^{1, p}$ and therefore $\mathcal{N}_{\lambda} \subset \mathcal{A}$. Furthermore, by (5.31), $\Phi_{\lambda}(u) \geq 0$ for any $(u, v) \in \mathcal{N}_{\lambda}$. But then

$$
\inf \left\{\Phi_{\lambda}(u, v):(u, v) \in \mathcal{N}_{\lambda}\right\}=0 .
$$

Indeed, since $\lambda>\lambda_{1, q}$, it is not hard to find $\phi_{0} \in \dot{W}_{0}^{1, q}$ and a sequence $\left(v_{m}\right) \subset \dot{W}_{0}^{1, q}$ such that $v_{m} \rightarrow \phi_{0}$ in $W_{0}^{1, q}$ as $m \rightarrow+\infty, Q_{\lambda}\left(\phi_{0}\right)=0, Q_{\lambda}\left(v_{m}\right)>0, m=1,2, \ldots$, and $Q_{\lambda}\left(v_{m}\right) \rightarrow 0$ as $m \rightarrow+\infty$. Hence, under assumption (5.32) we can find $\psi_{0} \in \dot{W}_{0}^{1, p}$ such that $F\left(\psi_{0}, \phi_{0}\right)>0$. Then $P_{\lambda}\left(\psi_{0}\right)>0$ and $F\left(\psi_{0}, v_{m}\right) \geq c_{0}>0$ for sufficient large $m$, since $v_{m} \rightarrow \phi_{0}$ in $W_{0}^{1, q}$. Setting now $u_{m}=\psi_{0}$ for $m=1,2, \ldots$ we get by (5.31) that $\Phi_{\lambda}\left(u_{m}, v_{m}\right) \rightarrow 0$ as $m \rightarrow+\infty$ and thus (5.33) follows. Furthermore, by this construction we got a minimizing sequence with unsatisfactory properties. Indeed, since $\frac{\alpha}{p}+\frac{\beta}{q}>1$, one of the following must hold $p<\alpha$ or/and $q<\beta$. Hence (5.29), (5.30) imply that if $p<\alpha$, then $t_{m}:=t\left(u_{m}, v_{m}\right) \rightarrow 0$, 
$s_{m}:=s\left(u_{m}, v_{m}\right) \rightarrow+\infty$ and if $p \geq \alpha$, then $t_{m} \rightarrow 0, s_{m} \rightarrow+0$. Thus in the first case we have unbounded above minimizing sequence $\left(t_{m} u_{m}, s_{m} v_{m}\right)$ whereas in the second case this sequence converges to zero.

Here we do not consider the application of the scalar NMM to (5.21). One of the reasons is that we could not find a suitable formula for determining or evaluation the extremal value like $\lambda_{\max }^{s c, *}$. Another difficulty lies in finding of solutions of the corresponding scalar minimization problem (2.7). However, by (4.5), (4.6) we have $\left(\lambda_{\text {min }}^{s c}, \lambda_{\text {max }}^{s c, *}\right) \subseteq\left(\lambda_{\min }, \lambda_{\text {max }}^{*}\right)$. Thus we should not expect that the scalar NMM will provide the better results than by the vector NMM (5.24).

\section{Multiplicity Result}

Nehari manifold methods is often used to prove the existence of multiple solutions, see e.g. [10, 13, 23, 35. In this Section, we show how to obtain such type of results using NG-Rayleigh's quotient.

We will study (2.1) using the scalar NMM. First we prove some general result and then we give an example to illustrate it. In what follows, we always assume that $D_{u} G(u)(u) \neq 0$ for all $u \in W \backslash 0_{n}$ so that $\mathcal{W}=W \backslash 0_{n}$. We will suppose that NG-Rayleigh's quotient $r(u)$ satisfies the following conditions: for $u \in W \backslash 0_{n}$

(a) $\tilde{r}^{s c}(t, u):=r(t u)$ attains its global maximum at a unique point $t_{u, \max }>0$ : $r\left(t_{u, \max } u\right)=\max _{t>0} r(t u)$ so that $\partial r\left(t_{u, \max } u\right) / \partial t=0$ and

$$
\partial r(t u) / \partial t>0 \text { for } 0<t<t_{u, \max }, \partial r(t u) / \partial t<0 \text { for } t>t_{u, \max } .
$$

Note that if assumption (a) holds for every $u \in W \backslash 0_{n}$, then (A) and (S) follow (see Remark 4.3). Furthermore, (a) implies that for every $u \in W \backslash 0_{n}$, there exist the limits

- $r(t u) \rightarrow \tilde{r}^{s c}(0, u)$ as $t \rightarrow 0$, where $-\infty \leq \tilde{r}^{s c}(0, u)<+\infty$

- $r(t u) \rightarrow \tilde{r}^{s c}(\infty, u)$ as $t \rightarrow+\infty$, where $-\infty \leq \tilde{r}^{s c}(\infty, u)<+\infty$.

Consider $\lambda_{\text {max }}^{*}=\inf _{u \in W \backslash 0_{n}} \sup _{t>0} r(t u)=\inf _{u \in W \backslash 0_{n}} r\left(t_{u, \max } u\right)$ and

$$
\lambda_{\text {min }}^{\partial}=\sup _{u \in W \backslash 0_{n}} \max \left\{\tilde{r}^{s c}(0, u), \tilde{r}^{s c}(\infty, u)\right\} .
$$

Note that (a) implies

$$
\lambda_{\text {min }}^{*}=\sup _{u \in W \backslash 0_{n}} \inf _{t>0} r(t u) \leq \lambda_{\min }^{\partial}
$$

Let us introduce the following sets

$$
\begin{array}{ll}
\mathcal{N}_{\lambda}^{1}:=\left\{u \in W \backslash 0_{n}:\right. & \left.r(u)=\lambda, \quad \partial r(t u) /\left.\partial t\right|_{t=1}<0\right\} \\
\mathcal{N}_{\lambda}^{2}:=\left\{u \in W \backslash 0_{n}:\right. & \left.r(u)=\lambda, \quad \partial r(t u) /\left.\partial t\right|_{t=1}>0\right\} .
\end{array}
$$

Obviously, $\mathcal{N}_{\lambda}^{1} \cap \mathcal{N}_{\lambda}^{2}=\emptyset$ for any $\lambda<\lambda_{\text {max }}^{*}$, and $\mathcal{N}_{\lambda}^{1} \neq \emptyset, \mathcal{N}_{\lambda}^{2} \neq \emptyset$ for any $\lambda \in$ $\left(\lambda_{\text {min }}^{\partial}, \lambda_{\text {max }}^{*}\right)$ provided $\lambda_{\text {min }}^{\partial}<\lambda_{\text {max }}^{*}$. Moreover, for $\lambda \in \mathbb{R}, \mathcal{N}_{\lambda}^{1} \cup \mathcal{N}_{\lambda}^{2}=\mathcal{N}_{\lambda}^{s c}$ if $\mathcal{N}_{\lambda}^{s c} \neq \emptyset$. Thus, when $\mathcal{N}_{\lambda}^{2} \neq \emptyset$ and $\mathcal{N}_{\lambda}^{1} \neq \emptyset$ one may split minimization problem (3.3) into the two following

$$
\begin{array}{ll}
\hat{\Phi}_{\lambda}^{1}:=\min \left\{\Phi_{\lambda}(u):\right. & \left.u \in \mathcal{N}_{\lambda}^{1}\right\} \\
\hat{\Phi}_{\lambda}^{2}:=\min \left\{\Phi_{\lambda}(u):\right. & \left.u \in \mathcal{N}_{\lambda}^{2}\right\} .
\end{array}
$$

Suppose in addition to the assumptions already made: 
(b) For any sequence $\left(v_{m}\right) \subset W \backslash 0_{n}$ such that $\left\|v_{m}\right\|=1, m=1,2, \ldots$ and which is weakly separated from $0_{n} \in W$, the set of functions $\left(\psi_{m}(t):=r\left(t v_{m}\right)\right)_{m=1}^{\infty}$ is bounded in $C^{1}[\sigma, T]$ for every $\sigma, T$ such that $T>\sigma>0$.

(c) For any sequence $\left(v_{m}\right) \subset W \backslash 0_{n},\left\|v_{m}\right\|=1, m=1,2, \ldots$ there exists $\delta>0$ such that $t_{v_{m}, \max }>\delta$ for all $m=1,2, \ldots$.

(d) If $\left(s_{m} v_{m}\right) \subset \mathcal{N}_{\lambda}^{s c}, \lambda \in \mathbb{R}$ such that $\left\|v_{m}\right\|_{W}=1, v_{m} \rightarrow 0_{n}$ weakly in $W$ and $\inf _{m} s_{m}>\delta_{0}>0$. Then $s_{m} \rightarrow+\infty$.

Theorem 6.1. Suppose $W$ is a reflexive Banach space, $\Phi_{\lambda} \in C^{1}(\dot{W}, \mathbb{R}), \nabla_{t} \tilde{\Phi}_{\lambda} \in$ $C^{1}\left(\left(\dot{\mathbb{R}}^{+}\right)^{n} \times \dot{W}, \mathbb{R}^{n}\right), \Phi_{\lambda}\left(0_{n}\right)=0, D_{u} G(u)(u)>0, \forall u \in W \backslash 0_{n}$, (a)- (d) hold and the following conditions are fulfilled:

(1) $\Phi_{\lambda}(u) \rightarrow+\infty$ as $\|u\| \rightarrow \infty, u \in \mathcal{N}_{\lambda}^{s c}$.

(2) $\Phi_{\lambda}(u)$ and $r(u)$ are (sequentially) weakly lower semi-continuous functionals on $W$.

Assume $\lambda_{\text {min }}^{\partial}<\lambda_{\text {max }}^{*}$. Then for every $\lambda \in\left(\lambda_{\text {min }}^{\partial}, \lambda_{\text {max }}^{*}\right)$ equation (2.1) possess two distinct solutions $u_{\lambda}^{1}, u_{\lambda}^{2} \in W \backslash 0_{n}$ such that $u_{\lambda}^{1}$ is a minimizer of (6.4) and $u_{\lambda}^{2}$ is a minimizer of (6.5). Furthermore, $\left.\partial^{2} \Phi_{\lambda}\left(t u_{\lambda}^{1}\right) \partial t^{2}\right|_{t=1}<0,\left.\partial^{2} \Phi_{\lambda}\left(t u_{\lambda}^{2}\right) \partial t^{2}\right|_{t=1}>0$, $\Phi_{\lambda}\left(u_{\lambda}^{2}\right) \equiv \hat{\Phi}_{\lambda}^{2}<\Phi_{\lambda}(0)$ and $u_{\lambda}^{2}$ is the ground state of (2.1).

Proof. First let us show $\hat{\Phi}_{\lambda}^{2}<0$ for $\lambda \in\left(\lambda_{\text {min }}^{\partial}, \lambda_{\text {max }}^{*}\right)$. Choose $u \in \mathcal{N}_{\lambda}^{2}$. Then since $r(u)=\lambda$, condition (a) implies that $r(t u)<\lambda$ for all $t \in(0,1)$. Consequently by Corollary 3.1 $\partial \Phi_{\lambda}(t u) / \partial t<0$ for all $t \in(0,1)$ and therefore $0=\Phi_{\lambda}(0 u)>$ $\Phi_{\lambda}(u) \geq \hat{\Phi}_{\lambda}^{2}$.

Let $\left(u_{m}\right)$ be a minimizing sequence $\left(u_{m}\right)$ of (6.4) or (6.5), where $\lambda \in\left(\lambda_{\min }^{\partial}, \lambda_{\max }^{*}\right)$. From (1) it follows that $\left(u_{m}\right)$ is bounded in $W$. Since $W$ is reflexive Banah space, by the Eberlein-Smulian theorem we may assume that $u_{m} \rightarrow u_{0}$ weakly in $W$ as $m \rightarrow \infty$ for some $u_{0} \in W$. Write $u_{m}=s_{m} v_{m}$ where $s_{m}=\left\|u_{m}\right\|, m=1,2, \ldots,$. Then $\left(s_{m}\right)$ is bounded above and we may assume $s_{m} \rightarrow s_{0}, v_{m} \rightarrow v_{0}$ weakly in $W$ as $m \rightarrow \infty$ for some $s_{0} \geq 0$ and $v_{0} \in W$.

Let us show that $s_{0} \neq 0$ and $v_{0} \neq 0_{n}$. Consider first (6.4). In view of (a), we have $s_{m}>t_{v_{m}, \max }$. Therefore by (c), $\inf _{m} s_{m}>\delta_{0}>0$ for some $\delta_{0}>0$ and thus $s_{0} \neq 0$. Suppose, contrary to our claim, that $v_{m} \rightarrow 0_{n}$ weakly in $W$. Then (d) implies $s_{m} \rightarrow+\infty$. Hence we get a contradiction and thus $v_{0} \neq 0_{n}$.

Let now $\left(u_{m}=s_{m} v_{m}\right)$ be a minimizing sequence of (6.5). Assume $s_{m} \rightarrow 0$. Then $\left\|u_{m}\right\| \rightarrow 0$ and $\Phi_{\lambda}\left(u_{m}\right) \rightarrow 0$. However, $\hat{\Phi}_{\lambda}^{2}<0$ and we obtain a contradiction. Thus $s_{0} \neq 0$. Suppose, contrary to our claim, that $v_{m} \rightarrow 0_{n}$ weakly in $W$. Then since $\inf _{m} s_{m}>\delta_{0}^{\prime}>0$ for some $\delta_{0}^{\prime}>0$, we get by (d) a contradiction. Thus in this case we have also $v_{0} \neq 0_{n}$.

Thus the set $B:=\left(v_{m}\right)$ is bounded above and weakly separated from $0_{n} \in W$. Then (b) implies that the set of functions $\left(\psi_{m}(t):=r\left(t v_{m}\right)\right)_{m=1}^{\infty}$ is bounded in $C^{1}[\sigma, T]$ for any $T>\sigma>0$. Consequently by the Arzelá-Ascoli compactness criterion we can assume that

$$
\psi_{m}(t) \rightarrow \psi(t) \text { in } C[\sigma, T], \quad \forall T, \sigma \text { s.t. } T>\sigma>0,
$$

for some limit function $\psi \in C(0,+\infty)$.

Since $s_{0}>0$, 6.6.6) yields

$$
r\left(t u_{m}\right)=r\left(t\left(s_{m} v_{m}\right)\right) \rightarrow \psi\left(t s_{0}\right)=: \hat{r}(t) \text { as } m \rightarrow \infty, \quad \text { in } C[\sigma, T],
$$


for any $T>\sigma>0$. Furthermore, by weak lower semi-continuity of $r(t u)$, for every $t>0$, we have

$$
r\left(t u_{0}\right) \leq \liminf _{m \rightarrow \infty} r\left(t u_{m}\right) .
$$

This and (6.7) yield that for $t \geq 0$

$$
r\left(t u_{0}\right) \leq \hat{r}(t) .
$$

Proposition 6.2. $u_{0}$ satisfies $\partial r\left(t u_{0}\right) /\left.\partial t\right|_{t=1}>0$ for (6.5) and $\partial r\left(t u_{0}\right) /\left.\partial t\right|_{t=1}<0$ for (6.4).

Proof. We prove the assertion only for problem (6.5); problem (6.4) can be handled in a similar way.

Since $r\left(t u_{m}\right) \leq \lambda$ for $t \in(0,1]$, 6.7) implies $\hat{r}(t) \leq \lambda$ for $t \in(0,1]$. Suppose, contrary to our claim, that $\partial r\left(u_{0} t\right) /\left.\partial t\right|_{t=1}<0$. Then due to property (a) one has $t_{u_{0}}<1$, where $r\left(t_{u_{0}} u_{0}\right)=\max _{t>0} r\left(t u_{0}\right)$. Since $\hat{r}(t) \leq \lambda$ for $t \in(0,1]$ we deduce from (6.9) that $r\left(t_{u_{0}} u_{0}\right) \leq \lambda$. But by the assumption $\lambda<\lambda_{\text {max }}^{*} \leq \max _{t>0} r\left(t u_{0}\right)$, a contradiction.

Now let us conclude the proof of the theorem. By weak lower semi-continuity of $\Phi_{\lambda}$ we have

$$
-\infty<\Phi_{\lambda}\left(u_{0}\right) \leq \liminf _{m \rightarrow \infty} \Phi_{\lambda}\left(u_{m}\right)=\hat{\Phi}_{\lambda}^{ \pm} .
$$

Hence, for the existence of the minimizers $u_{\lambda}^{1}$ and $u_{\lambda}^{2}$ of (6.4) and (6.5), respectively, it is sufficient to show that $r\left(u_{0}\right)=\lambda$.

Suppose, contrary to our claim, that $r\left(u_{0}\right)<\lambda$. First consider problem 6.5. . By Proposition 6.2 $\partial r\left(t u_{0}\right) /\left.\partial t\right|_{t=1}>0$. Consequently property (a) and the assumption $\lambda<\lambda_{\max }^{*} \leq r\left(t_{u_{0}} u_{0}\right)=\max _{t>0} r\left(t u_{0}\right)$ yield that there is $t_{1} \in\left(1, t_{u_{0}}\right)$ such that $r\left(t_{1} u_{0}\right)=\lambda$ and $\partial r\left(t u_{0}\right) /\left.\partial t\right|_{t=t_{1}}>0$. Moreover, since $r\left(t u_{0}\right)<\lambda$ for $t \in\left[1, t_{1}\right)$, Corollary 3.1 implies that $\partial \Phi_{\lambda}(t u) / \partial t<0$ for $t \in\left[1, t_{1}\right)$. Consequently

$$
\Phi_{\lambda}\left(t_{1} u_{0}\right)<\Phi_{\lambda}\left(u_{0}\right) \text {. }
$$

Thus by (6.10) we have $\Phi_{\lambda}\left(t_{1} u_{0}\right)<\hat{\Phi}_{\lambda}$ and since $t_{1} u_{0} \in \mathcal{N}_{\lambda}^{2}$, we obtain a contradiction.

Consider now problem (6.4). Since $\partial r\left(t u_{0}\right) /\left.\partial t\right|_{t=1}<0, \lambda<\lambda_{\text {max }}^{*}$, assumption (a) implies that there is $t_{2}<1$ such that $r\left(t_{2} u_{0}\right)=\lambda$ and $\partial r\left(t u_{0}\right) /\left.\partial t\right|_{t=t_{2}}<0$. Note that by the weak lower semi-continuity of $\Phi_{\lambda}$

$$
\Phi_{\lambda}\left(t_{2} u_{0}\right) \leq \liminf _{m \rightarrow \infty} \Phi_{\lambda}\left(t_{2} u_{m}\right) .
$$

Since (6.8) and $r\left(t_{2} u_{0}\right)=\lambda, r\left(u_{m}\right)=\lambda$, assumption (a) implies that $r\left(t u_{m}\right)>\lambda$ for $t \in\left(t_{2}, 1\right]$ and sufficiently large $m$. Then Corollary 3.1 implies that $\partial \Phi_{\lambda}\left(t u_{m}\right) / \partial t>$ 0 for $t \in\left(t_{2}, 1\right]$ and sufficiently large $m$. Consequently, $\Phi_{\lambda}\left(t_{2} u_{m}\right)<\Phi_{\lambda}\left(u_{m}\right)$ and (6.11) yields

$$
\Phi_{\lambda}\left(t_{2} u_{0}\right) \leq \liminf _{m \rightarrow \infty} \Phi_{\lambda}\left(t_{2} u_{m}\right) \leq \liminf _{m \rightarrow \infty} \Phi_{\lambda}\left(u_{m}\right)=\hat{\Phi}_{\lambda}^{1} .
$$

Assume $\Phi_{\lambda}\left(t_{2} u_{0}\right)=\hat{\Phi}_{\lambda}^{-}$, then taking into account that $t_{2} u_{0} \in \mathcal{N}_{\lambda}^{1}$ we see that $t_{2} u_{0}$ is a minimizer of (6.4) and we get the required. If $\Phi_{\lambda}\left(t_{2} u_{0}\right)<\hat{\Phi}_{\lambda}^{1}$, then we obtain a contradiction and therefore $r\left(u_{0}\right)=\lambda$. This completes the proof of the existence of the minimizers $u_{\lambda}^{1}$ and $u_{\lambda}^{2}$.

Now in view of (6.1), Theorem 4.6 yields that $u_{\lambda}^{1}$ and $u_{\lambda}^{2}$ satisfy (2.1). From Corollary 3.2 it follows that $\left.\partial^{2} \Phi_{\lambda}\left(t u_{\lambda}^{1}\right) \partial t^{2}\right|_{t=1}<0,\left.\partial^{2} \Phi_{\lambda}\left(t u_{\lambda}^{2}\right) \partial t^{2}\right|_{t=1}>0$. To conclude the proof, it remains to show that $u_{\lambda}^{2}$ is the ground state of (2.1). Assumption 
(a) and $\lambda \in\left(\lambda_{\text {min }}^{\partial}, \lambda_{\text {max }}^{*}\right)$ yield that the equation $\partial \Phi_{\lambda}\left(s u_{\lambda}^{1}\right) / \partial s=0$ has precisely two solutions $s_{\min }<1$ and $s_{\max }=1$ such that $\partial^{2} \Phi_{\lambda}\left(s u_{\lambda}^{1}\right) /\left.\partial s^{2}\right|_{s=s_{\min }}>0$ and $\partial^{2} \Phi_{\lambda}\left(s u_{\lambda}^{2}\right) /\left.\partial s^{2}\right|_{s=1}<0$. But then $\Phi_{\lambda}\left(u_{\lambda}^{1}\right)>\Phi_{\lambda}\left(s_{\min } u_{\lambda}^{1}\right) \geq \hat{\Phi}_{\lambda}^{2} \equiv \Phi_{\lambda}\left(u_{\lambda}^{2}\right)$. Thus $u_{\lambda}^{2}$ is the minimizer of $\Phi_{\lambda}:=\min \left\{\Phi_{\lambda}(u): u \in \mathcal{N}_{\lambda}\right\}$ and we get the required.

We emphasize that the value $\lambda_{\min }^{\partial}$ has been used above only in order to allocate the values $\lambda$ in $\left(\lambda_{\text {min }}^{\partial}, \lambda_{\text {max }}^{*}\right)$ such that $\mathcal{N}_{\lambda}^{1} \neq \emptyset, \mathcal{N}_{\lambda}^{2} \neq \emptyset$. In fact, the above proof of Theorem 6.1 can be easily adapted to other assumptions on the behaviour of $r(t u)$ at $t \rightarrow 0$ and $t \rightarrow \infty$. In particular, let us assume that for all $u \in W \backslash 0_{n}$ there holds

$$
r(t u) \rightarrow 0 \text { as } t \rightarrow 0 \text { and } r(t u) \rightarrow-\infty \text { as } t \rightarrow \infty .
$$

It easy to see that then $\lambda_{\text {min }}^{\partial}=0$ and $\lambda_{\text {min }}^{*}=-\infty$. Moreover, now we have $\mathcal{N}_{\lambda}^{1} \neq \emptyset$ for all $\lambda<\lambda_{\max }^{*}$. It is easily seen that the above proof of the existence of the minimizer $u_{\lambda}^{1}$ of (6.4) remains valid for all $\lambda<\lambda_{\text {max }}^{*}$, provided (6.12) is satisfied. Thus we have

Corollary 6.3. Suppose the assumptions of Theorem 6.1 and (6.12) hold. Then for every $\lambda<\lambda_{\text {max }}^{*}$ there exists a minimizer $u_{\lambda}^{1}$ of (6.4) which satisfies equation (2.1). Furthermore, $\left.\partial^{2} \Phi_{\lambda}\left(t u_{\lambda}^{1}\right) \partial t^{2}\right|_{t=1}<0$ and $u_{\lambda}^{1}$ is the ground state of (2.1) if $\lambda \leq 0$.

Remark 6.4. We stress that the obtained (in Theorem 6.1, Corollary 6.3) solutions $u_{\lambda}^{1}, u_{\lambda}^{2}$ belong $W \backslash 0_{n}$. This means that in the case $n>1$ it is possible that $u_{i, \lambda}^{1}=0$, $u_{j, \lambda}^{2}=0$ for some $i, j=1,2 \ldots n$ whereas $u_{\lambda}^{1}, u_{\lambda}^{2} \not \equiv 0_{n}$ (see also Remark 2.4).

\subsection{Multiplicity nonnegative solutions for problems with a general convex-concave type nonlinearity}

In this subsection, using Theorem 6.1 we obtain a result on the existence of multiple sign-constant solutions for problems with a general convex-concave type nonlinearity and $p$-Laplacian.

Consider the following boundary value problem

$$
\left\{\begin{array}{l}
-\Delta_{p} u=\lambda|u|^{q-2} u+f(x, u), x \in \Omega, \\
\left.u\right|_{\partial \Omega}=0,
\end{array}\right.
$$

where $\Omega$ is a bounded domain in $\mathbb{R}^{N}$ with smooth boundary $\partial \Omega, N \geq 1, \Delta_{p}(\cdot)=$ $\operatorname{div}\left(|\nabla(\cdot)|^{p-2} \nabla(\cdot)\right)$ is the p-Laplacian, $1<q<p<+\infty, f: \Omega \times \mathbb{R} \rightarrow \mathbb{R}$ is a Carathéodory function such that $f(x, 0)=0, f(x, \cdot) \in C(\mathbb{R}, \mathbb{R}) \cap C^{1}(\mathbb{R} \backslash 0, \mathbb{R})$ a.a. $x \in \Omega$, with primitive $F(x, u)=\int_{0}^{u} f(x, s) d s$.

We will suppose that $f$ satisfies the following conditions:

$\left(1^{o}\right) \exists \gamma_{1}, \gamma_{2} \in\left(p, p^{*}\right), \gamma_{1} \leq \gamma_{2}$ such that

$0<s \frac{\partial}{\partial s} f(x, s) \leq g_{1}(x)|s|^{\gamma_{1}-2}+g_{2}(x)|s|^{\gamma_{2}-2}, \quad s \in \mathbb{R}, \quad$ a.a. $x \in \Omega$,

where $g_{i} \in L^{\beta_{i}}(\Omega), g_{i} \geq 0, \beta_{i}>p^{*} /\left(p^{*}-\gamma_{i}\right)$ if $N>p$ and $\beta_{i}>1$ if $N \leq p, i=1,2$.

$\left(2^{o}\right) \quad \exists \theta>p, R_{1}>0: 0<\theta F(x, s) \leq f(x, s) s$, a.a. $x \in \Omega$, if $|s| \geq R_{1}$ 
$\left(3^{\circ}\right)$ for $\forall u \in \dot{\mathbb{R}}$ and a.a. $x \in \Omega, \frac{\partial}{\partial s}\left(s^{1-q} f(x, s u)\right) u$ is a monotone increasing function on $(0,+\infty)$.

Example of $f$ satisfying $\left(1^{o}\right)-\left(3^{o}\right)$ is as follows: $f(x, s)=\sum_{i=1}^{l} f_{i}(x)|s|^{\gamma_{i}-2} s+$ $\psi(s), s \in \mathbb{R}$, where $p<\gamma_{1} \leq \ldots \leq \gamma_{l}<p^{*}, \psi \in C_{0}^{1}(\mathbb{R})$ such that $\psi(t)=0$ if $|t|<R_{0}$ for some $R_{0}>0, f_{i} \geq 0$ on $\Omega, f_{i} \in L^{\beta_{i}}(\Omega), \beta_{i}$ is defined as in $\left(1^{o}\right), i=1, \ldots, l$.

By a solution of (6.13) we shall mean a weak solution $u \in W:=W_{0}^{1, p}(\Omega)$. Problem (6.13) has a variational form with the Euler-Lagrange functional

$$
\Phi_{\lambda}(u)=\frac{1}{p} \int|\nabla u|^{p} d x-\lambda \frac{1}{q} \int|u|^{q} d x-\int F(x, u) d x .
$$

Consider NG-Rayleigh's quotient

$$
r(u)=\frac{\int|\nabla u|^{p} d x-\int f(x, u) u d x}{\int|u|^{q} d x}, \quad u \in W \backslash 0
$$

and the corresponding fibered map

$$
r(t u)=\frac{t^{p-q} \int|\nabla u|^{p} d x-t^{1-q} \int f(x, t u) u d x}{\int|u|^{q} d x}, u \in W \backslash 0, t>0 .
$$

Note that $\left(1^{\circ}\right)$ implies

$$
0<f(x, s) \leq g_{1}^{\prime}(x)|s|^{\gamma_{1}-1}+g_{2}^{\prime}(x)|s|^{\gamma_{2}-1} \quad \text { a.a. } x \in \Omega,
$$

where $g_{i}^{\prime}=g_{i} /\left(\gamma_{i}-1\right), i=1,2$. By Sobolev's embedding theorem and Holder's inequality for $u \in W$ and $i=1,2$ one has

$$
\left.\left.\left|\int_{\Omega} g_{i}^{\prime}(x)\right| u\right|^{\gamma_{i}} d x|\leq C||u|\right|^{\gamma_{i} / p^{*}}\left(\int_{\Omega}\left|g_{i}^{\prime}(x)\right|^{p^{*} /\left(p^{*}-\gamma_{i}\right)} d x\right)^{\frac{\left(p^{*}-\gamma_{i}\right)}{p^{*}}},
$$

where $C<+\infty$. Hence, $\Phi_{\lambda}$ and $r$ are well defined on $W$ and $W \backslash 0$, respectively.

Consider the extremal value

$$
\lambda_{\max }^{*}=\inf _{u \in W \backslash 0} \sup _{t>0} \frac{t^{p-q} \int|\nabla u|^{p} d x-t^{1-q} \int f(x, t u) u d x}{\int|u|^{q} d x} .
$$

We prove the following

Theorem 6.5. Assume $1<q<p<+\infty$ and $\left(1^{\circ}\right)-\left(3^{\circ}\right)$ hold. Then $0<\lambda_{\max }^{*}$ and for any $\lambda<\lambda_{\text {max }}^{*}$, problem (6.13) admits a pair of non-trivial weak solutions $u_{\lambda}^{1,+} \geq 0 \geq u_{\lambda}^{1,-}$. Furthermore, when $\lambda \in\left(0, \lambda_{\text {max }}^{*}\right)$ equation (6.13) has a second pair of non-trivial weak solutions $u_{\lambda}^{2,+} \geq 0 \geq u_{\lambda}^{2,-}$. Moreover,

(1) $\left.\partial^{2} \Phi_{\lambda}\left(t u_{\lambda}^{1, \pm}\right) \partial t^{2}\right|_{t=1}<0,\left.\partial^{2} \Phi_{\lambda}\left(t u_{\lambda}^{2, \pm}\right) \partial t^{2}\right|_{t=1}>0, \Phi_{\lambda}\left(u_{\lambda}^{2, \pm}\right)<0$;

(2) if $\lambda \leq 0$, then one of the solutions $u_{\lambda}^{1,+}$ or $u_{\lambda}^{1,-}$ is a ground state of (6.13);

(3) if $\lambda \in\left(0, \lambda_{\max }^{*}\right)$, then one of the solutions $u_{\lambda}^{2,+}$ or $u_{\lambda}^{2,-}$ is a ground state of (6.13).

Remark. Similar result on the existence of multiple sign-constant solutions for problems with a general convex-concave type nonlinearity has been obtained in [2, 3, 31. However, our assumptions on function $f(x, s)$ are different from that were made in [2, 3, 31]. In particular, in $\left(1^{\circ}\right)$ the functions $g_{i}, i=1,2$ permit be unbounded above, which causes difficulties in application of the super-sub solution method (cf. [2, 3, 31]). Furthermore, the presence of $p$-Laplacian with $p \neq 2$ in (6.13) can complicate the application of mountain pass theorem in order interval (cf [31]). 
Proof. We will obtain the proof applying Theorem 6.1 and Corollary 6.3. Observe that (6.15) satisfies (a) for any $u \in W \backslash 0$. Indeed, compute

$$
\partial_{t} r(t u)=\frac{(p-q) t^{p-q-1} \int|\nabla u|^{p} d x-\int \partial_{t}\left(t^{1-q} f(x, t u)\right) u d x}{\int|u|^{q} d x} .
$$

It can easily be checked that $\left(1^{\circ}\right)$ and (6.17) yield $\int \partial_{t}\left(t^{1-q} f(x, t u)\right) u d x / t^{p-q-1} \rightarrow 0$ as $t \rightarrow 0$ for any $u \in W$. Hence by $\left(3^{\circ}\right)$ we get (a). Furthermore, it follows that (6.16) satisfies condition (6.12) of Corollary 6.3.

Let us show that (b) holds. Assume that $\left(u_{m}\right) \subset W \backslash 0$ such that $\left\|u_{m}\right\|=1$, $m=1,2, \ldots$, and the set $\left(u_{m}\right)_{m=1}^{\infty}$ is weakly separated from $0 \in W$. Since $\left(u_{m}\right)$ is bounded in $W$ and $W$ is the reflexive Banach space, by the Eberlein-Smulian theorem we may assume that $u_{m} \rightarrow u_{0}$ weakly in $W$ for some $u_{0} \in W$. Furthermore, by Sobolev's embedding theorem $\left\|u_{m}\right\|_{L^{d}}<C_{1}<+\infty$ for $m=1,2, \ldots, 1 \leq d \leq p^{*}$, and $u_{m} \rightarrow u_{0}$ in $L^{d}(\Omega)$ for $d<p^{*}$. This implies that there exists $\delta_{0}>0$ such that $\int\left|u_{m}\right|^{q} d x>\delta_{0}$ for all $m=1,2, \ldots$. Indeed, if we assume the converse, $u_{m_{j}} \rightarrow 0$ in $L^{q}(\Omega)$ for some subsequence $m_{j} \rightarrow+\infty$, then $u_{m_{j}} \rightarrow 0$ weakly in $W$ which contradicts the assumption that $\left(u_{m}\right)_{m=1}^{\infty}$ is weakly separated from $0 \in W$. Hence by $\left(1^{\circ}\right)$, 6.17) and (6.18) we have: for any $\mathrm{t}>0$

$$
\left|r\left(t u_{m}\right)\right| \leq \delta_{0}^{-1}\left(t^{p-q}+t^{1-q} \int\left|f\left(x, t u_{m}\right) u_{m}\right| d x\right) \leq C_{2} t^{p-q}+C_{3} t^{\gamma_{2}-q},
$$

and

$$
\begin{aligned}
\left|\partial_{t} r\left(t u_{m}\right)\right| & \leq \delta_{0}^{-1}\left((p-q) t^{p-q-1}+(q-1) t^{-q} \int\left|f\left(x, t u_{m}\right) u_{m}\right| d x+\right. \\
& \left.t^{1-q} \int\left|\partial_{t} f\left(x, t u_{m}\right) u_{m}\right| d x\right) \leq C_{4} t^{p-q-1}+C_{5} t^{\gamma_{2}-q-1}
\end{aligned}
$$

where $C_{2}, \ldots, C_{5}$ do not depend on $t>0$ and $m=1,2, \ldots$. Thus we get (b).

Let us verify $(\mathbf{c})$. Denote $t_{m}:=t_{v_{m}, \max }$ where $\left\|v_{m}\right\|=1, m=1,2, \ldots$ In view of (6.20) we have

$$
(p-q) t_{m}^{p-q-1}-\int \partial_{t}\left(t_{m}^{1-q} f\left(x, t_{m} v_{m}\right)\right) v_{m} d x=0 .
$$

Hence using $\left(1^{\circ}\right)$, 6.17) and (6.18) we get

$$
(p-q) t_{m}^{p-q-1}-c_{1} t_{m}^{\gamma_{2}-q-1}-c_{2} t_{m}^{\gamma_{1}-q-1} \leq 0,
$$

which is impossible if $t_{m} \rightarrow 0$ since by the assumption $p<\gamma_{2} \leq \gamma_{1}$. Thus (c) holds.

Let us verify (d). Observe that (6.17) and (6.18) imply

$$
r(t u) \geq \frac{t^{p-q} \int|\nabla u|^{p} d x-C_{1}^{\prime} t^{\gamma_{2}-q}\|u\|^{\gamma_{2}}-C_{2}^{\prime} t^{\gamma_{1}-q}\|u\|^{\gamma_{1}}}{\|u\|^{q}},
$$

for $t>0, u \in W \backslash 0$. Assume $\left(s_{m} v_{m}\right) \subset \mathcal{N}_{\lambda}^{s c}, \lambda \in \mathbb{R}$ such that $\left\|v_{m}\right\|_{W}=1, v_{m} \rightarrow 0$ weakly in $W$ and $\inf _{m} s_{m}>\delta>0$. Then, as it has been shown above $v_{m} \rightarrow 0$ in $L^{d}(\Omega)$ for $d \in\left(1, p^{*}\right)$. Hence, since $\gamma_{2} \leq \gamma_{1}$, by (6.22) we have

$$
s_{m}^{\gamma_{1}-q} \geq \frac{\delta_{0}^{p-q}-\lambda\left\|v_{m}\right\|_{L^{q}}^{q}}{C_{1}^{\prime} \delta_{0}^{\gamma_{2}-\gamma_{1}}\left\|v_{m}\right\|_{L^{\gamma_{2}}}^{\gamma_{2}}+C_{2}^{\prime}\left\|v_{m}\right\|_{L^{\gamma_{1}}}^{\gamma_{1}}} \rightarrow \infty
$$

Thus (d) also holds. 
Let us now verify conditions (1), (2) of Theorem 6.1. For $u \in \mathcal{N}_{\lambda}$ we have

$$
\Phi_{\lambda}(u)=\frac{(\theta-p)}{p} \int|\nabla u|^{p} d x-\lambda \frac{(\theta-q)}{q} \int|u|^{q} d x-\int(\theta F(x, u)-f(x, u) u) d x .
$$

Hence $\left(2^{\circ}\right)$ and Sobolev's embedding theorem yield

$$
\Phi_{\lambda}(u) \geq \frac{(\theta-p)}{p}\|u\|^{p}-\frac{\lambda(\theta-q)}{q}\|u\|^{q},
$$

for $\|u\|>R_{1}$. Since $q<p$, this implies $\Phi_{\lambda}(u) \rightarrow+\infty$ as $\|u\| \rightarrow+\infty$ and thus condition (1) of Theorem 6.1 holds.

By the above, the functionals $\int F(x, u) d x, \int f(x, u) u d x, \int|u|^{q} d x$ are weakly continuous on $W$. Hence, since $\int|\nabla u|^{p} d x$ is a weakly lower semi-continuous functional on $W$ we derive that (6.14) and 6.15) satisfy (2) of Theorem 6.1.

Note that (6.22), $\left(1^{\circ}\right)$ and Sobolev's embedding theorem yield

$$
\begin{gathered}
\lambda_{\max }^{*} \geq \inf _{u \in W:\|v\|=1} \sup _{t>0} \frac{t^{p-q}\|v\|^{p}-c_{1}^{\prime} t^{\gamma_{2}-q}\|v\|^{\gamma_{2}}-C_{1}^{\prime} t^{\gamma_{1}-q}\|v\|^{\gamma_{1}}}{\|v\|^{q}}= \\
\max _{t>0}\left\{t^{p-q}-c_{1}^{\prime} t^{\gamma_{2}-q}-C_{1}^{\prime} t^{\gamma_{1}-q}\right\}>0 .
\end{gathered}
$$

for some $c_{1}^{\prime}, C_{1}^{\prime}>0$. Hence $\lambda_{\max }^{*}>0$. Thus, we see that the functional $\Phi_{\lambda}(u)$ satisfies all assumptions of Theorem 6.1 and Corollary 6.3

In order to obtain solutions $u_{\lambda}^{1,+} \geq 0 \geq u_{\lambda}^{1,-}$ and $u_{\lambda}^{2,+} \geq 0 \geq u_{\lambda}^{2,-}$, we truncate and reflect $f(x, u)$ as follows

$$
f^{ \pm}(x, u)=\left\{\begin{array}{l}
f(x, u) \quad \text { if } \quad \pm u \geq 0, \\
f(x,-u) \quad \text { if } \quad \pm u<0 .
\end{array}\right.
$$

Let $F^{ \pm}(x, u)$ denote the primitive of $f^{ \pm}(x, u)$ and consider the Euler-Lagrange functional

$$
\Phi_{\lambda}^{ \pm}(u)=\frac{1}{p} \int|\nabla u|^{p} d x-\lambda \frac{1}{q} \int|u|^{q} d x-\int F^{ \pm}(x, u) d x .
$$

Then as above $\Phi_{\lambda}^{ \pm}(u) \in C^{1}(\dot{W}, \mathbb{R}), \nabla_{t} \tilde{\Phi}_{\lambda}^{ \pm} \in C^{1}\left(\left(\dot{\mathbb{R}}^{+}\right)^{n} \times \dot{W}, \mathbb{R}^{n}\right)$ it satisfies all the assumptions of Theorem 6.1 and Corollary 6.3. Thus there exist weak solutions $u_{\lambda}^{1, \pm}, u_{\lambda}^{2, \pm} \in W_{0}^{1, p}(\Omega)$ of

$$
-\Delta_{p} u=\lambda|u|^{q-2} u+f^{ \pm}(x, u),
$$

for $\lambda<\lambda_{\text {max }}^{*}$ and $\lambda \in\left(0, \lambda_{\text {max }}^{*}\right)$, respectively. Since $\Phi_{\lambda}^{ \pm}(|u|)=\Phi_{\lambda}^{ \pm}(u)$ we may assume that the minimizers $u_{\lambda}^{1,+}, u_{\lambda}^{2,+}$ of $\hat{\Phi}_{\lambda}^{j,+}:=\min \left\{\Phi_{\lambda}^{+}(u): u \in \mathcal{N}_{\lambda}^{j}\right\}, j=$ 1,2 , respectively, are non-negative, whereas the minimizers $u_{\lambda}^{1,-}, u_{\lambda}^{2,-}$ of $\hat{\Phi}_{\lambda}^{j,-}:=$ $\min \left\{\Phi_{\lambda}^{-}(u): u \in \mathcal{N}_{\lambda}^{j}\right\}, j=1,2$, respectively, are non-positive. Now taking into account (6.25) we get that the functions $u_{\lambda}^{1, \pm}, u_{\lambda}^{2, \pm}$ in fact are weak solutions of the original problem (6.13). Finally, the assertions (1)-(3) of the theorem follow from Theorem 6.1 and Corollary 6.3.

Acknowledgement. The authors would like to acknowledge the considerable benefit obtained from discussions with V. Bobkov and D. Motreanu. 


\section{REFERENCES}

[1] Arnol'd, Vladimir I. Catastrophe theory. Springer Science \& Business Media, 1992.

[2] Ambrosetti, A., Azorero, J. G., \& Peral, I. Multiplicity results for some nonlinear elliptic equations. Journal of Functional Analysis, 137(1), 219-242 (1996).

[3] Ambrosetti, A., Brezis, H., \& Cerami, G. Combined effects of concave and convex nonlinearities in some elliptic problems. Journal of Functional Analysis, 122(2), 519-543 (1994).

[4] Alama, S., Tarantello, G. On semilinear elliptic problems with indefinite nonlinearities. Calculus Var. and Partial Differential Equations 1, 439-475, (1993)

[5] Anane, A. Etude des valeurs propres et de la résonance pour l'opérateur $p$-Laplacien, Thèse de doctorat, Université Libre de Bruxelles, Brussels, (1988)

[6] Berestycki, H., Capuzzo-Dolcetta, I., Nirenberg, L. Variational methods for indefinite superlinear homogeneous elliptic problems. NoDEA, 2, 553-572 (1995).

[7] Bobkov, V., \& Il'yasov, Y. Asymptotic behaviour of branches for ground states of elliptic systems. Electronic Journal of Differential Equations, 2013 (212), 1-21 (2013).

[8] Bobkov, V., \& Il'yasov, Y. Maximal existence domains of positive solutions for two-parametric systems of elliptic equations. arXiv preprint arXiv:1406.5275, (2014).

[9] Bobkov, V. Least energy nodal solutions for elliptic equations with indefinite nonlinearity. Electronic Journal of Qualitative Theory of Differential Equations. (2014).

[10] Bozhkov, Y.\& Mitidieri, E. Existence of multiple solutions for quasilinear systems via fibering method. J. Differ. Equations 190, No.1, 239-267 (2003).

[11] Brown, K. J., T.F. Wu. A fibering map approach to a potential operator equation and its applications. Differential and Integral Equations 22.11/12, 1097-1114 (2009).

[12] Díaz, J. I., Hernández, J., \& Il'yasov, Y. On the existence of positive solutions and solutions with compact support for a spectral nonlinear elliptic problem with strong absorption. Nonlinear Analysis: Theory, Methods \& Applications, 119, 484-500 (2015).

[13] Drábek, P., Pohozaev, S.I. Positive solution for the p-Laplacian: application of the fibering method. Proc. Roy. Soc. Edinb. Sect. A 127, 703-726 (1997).

[14] Drábek, P., and Jaroslav M. Methods of nonlinear analysis: applications to differential equations. Springer Science \& Business Media, 2013.

[15] Drábek,P., Krejci, P.\& Takac, P. Nonlinear differential equations. Chapman \& Hall/CRC Boca Raton, FL, (1999)

[16] Cherfils, L., \& Il'yasov, Y. On the stationary solutions of generalized reaction diffusion equations with p\&q-Laplacian. Communications on Pure and Applied Mathematics, 4(1), 9-22(2005).

[17] Richard Courant: Über die Eigenwerte bei den Differentialgleichungen der mathematischen Physik. In: Mathematische Zeitschrift. 7, Nr. 1-4, 1920, S. 1-57.

[18] Egorov, Y. V., \& Il'yasov, Y. (2007). On conformal invariants for elliptic systems with multiple critical exponents. Annals of Global Analysis and Geometry, 32(1), 39-66.

[19] Ernst Fischer: Über quadratische Formen mit reellen Koeffizienten. In: Monatshefte für Mathematik und Physik. 16, 1905, S. 234-249.

[20] Il'yasov, Y., Sari, N. . Solutions of minimal period for a Hamiltonian system with a changing sign potential. Communications on Pure and Applied Analysis (CPAA), 4(1), 175-185 (2005)

[21] Il'yasov, Y., Y. Egorov, Hopf boundary maximum principle violation for semilinear elliptic equations, Nonlinear Analysis: Theory, Methods \& Applications 72 (7-8), 3346-3355, (2010)

[22] Il'yasov, Y. On a procedure of projective fibering of functionals on Banach spaces, Proc. Steklov Inst. Math., 232, 150-156, (2001)

[23] Il'yasov, Y. Non-local investigation of bifurcations of solutions of non-linear elliptic equations, Izv. RAN. Ser. Mat., 66:6, (2002)

[24] Il'yasov,Y. On nonlocal existence results for elliptic equations with convex-concave nonlinearities, Nonlinear Analysis: Theory, Methods \& Applications, 61 (1-2), 211-236, (2005)

[25] Il'yasov, Y., Runst, T. Positive solutions for indefinite inhomogeneous Neumann elliptic problems, Electronic Journal of Differential Equations, 57, 1-21, (2003)

[26] Il'yasov, Y., \& Runst, T. On nonlocal calculation for inhomogeneous indefinite Neumann boundary value problems. Calculus of Variations and Partial Differential Equations, 22(1), 101-127, (2005). 
[27] Il'yasov, Y., Runst, T.,\& Youssfi, A. On the existence of pair positive-negative solutions for resonance problems. Nonlinear Analysis: Theory, Methods \& Applications, 70(10), 3461-3471 (2009).

[28] Il'yasov, Y. S., \& Takác, P. Optimal-regularity, Pohozhaev's identity, and nonexistence of weak solutions to some quasilinear elliptic equations. Journal of Differential Equations, 252(3), 2792-2822 (2012).

[29] Il'yasov, Y. S. On calculation of the bifurcations by the fibering approach. In Harmonic, Wavelet and P-adic Analysis, (N. M. Chuong, et al. eds., World Scientific Publishing, 141155 (2007).

[30] John, F. Extremum problems with inequalities as subsidiary conditions. In: Kurt Friedrichs, Otto Neugebauer, J. J. Stoker (Hrsg.): Studies and Essays. Courant Anniversary Volume, Wiley, 1948, p. 187-204, In: Traces and Emergence of Nonlinear Programming . Springer Basel (pp. 197-215) (2014).

[31] Li, S., \& Wang, Z. Q. Mountain pass theorem in order intervals and multiple solutions for semilinear elliptic Dirichlet problems. Journal d'Analyse Mathematique, 81(1), 373-396 (2000).

[32] Lindqvist, P. On the equation $\operatorname{div}\left(|\nabla u|^{p-2} \nabla u\right)+\lambda|u|^{p-2} u=0$, Proc. Am. Math. Soc. 109, No.1, 157-164, (1990)

[33] Nehari, Z. On a class of nonlinear second-order differential equations. Transactions of the American Mathematical Society, 101-123, (1960).

[34] Nehari, Z. Characteristic values associated with a class of nonlinear second-order differential equations, Acta Math. 105, 141-175, (1961)

[35] Ouyang, T. On the positive solutions of semilinear equations $\Delta u+\lambda u+h u^{p}=0$ on the compact manifolds Part II, Indiana Univ. Math. J., 40, No.3, 1083-1141 (1991).

[36] Pohozaev, S.I. On an approach to Nonlinear equations. Doklady Acad. Sci. USSR, 247, 13271331, (1979)

[37] Pohozaev, S.I. On the method of fibering a solution in nonlinear boundary value problems, Proc. Stekl. Ins. Math., 192, 157-173, (1990)

[38] Pohozaev, S. I. The fibering method in nonlinear variational problems. Pitman Research Notes in Mathematics Series : 35-88, (1997).

[39] Walter Ritz "Über eine neue Methode zur Lösung gewisser Variationsprobleme der mathematischen Physik" Journal für die Reine und Angewandte Mathematik, vol. 135, pages 1-61(1909).

[40] Szulkin, A., \& Weth, T. The method of Nehari manifold. Handbook of nonconvex analysis and applications, 597-632 (2010).

[41] Weber, H. Über die Integration der partiellen Differentialgleichungen. Math. Ann., 1: 1-366 (1869)

[42] Strutt, John William, and Baron Rayleigh. The theory of sound, 2nd ed.rev. and enl. Dover Publications, New York (1945).

[43] Wu, Tsung-Fang. The Nehari manifold for a semilinear elliptic system involving sign-changing weight functions. Nonlinear Analysis: Theory, Methods \& Applications 68.6: 1733-1745 (2008).

YAVDAT IL'YASOV

Institute of Mathematics of RAS, UfA, Russia

E-mail address: ilyasov02@gmail.com 